OPEN ACCESS

Edited by:

Djamel Drider,

Lille University of Science and Technology, France

Reviewed by:

Christian Magni,

Instituto de Biología Molecular y

Celular de Rosario (CONICET),

Argentina

Paloma López,

Centro de Investigaciones Biológicas

(CIB), Spain

Olivia McAuliffe,

Teagasc, The Irish Agriculture and Food Development Authority,

Ireland

*Correspondence: Beatriz Martínez bmf1@ipla.csic.es

Specialty section: This article was submitted to

Food Microbiology,

a section of the journal

Frontiers in Microbiology

Received: 29 June 2018 Accepted: 17 October 2018 Published: 05 November 2018

Citation:

López-González MJ, Escobedo S, Rodríguez A, Neves $A R$, Janzen $T$ and Martínez B (2018) Adaptive Evolution of Industrial Lactococcus lactis Under Cell Envelope Stress Provides Phenotypic Diversity.

Front. Microbiol. 9:2654. doi: 10.3389/fmicb.2018.02654

\section{Adaptive Evolution of Industrial Lactococcus lactis Under Cell Envelope Stress Provides Phenotypic Diversity}

\author{
María Jesús López-González", Susana Escobedo', Ana Rodríguez¹, A. Rute Neves², \\ Thomas Janzen ${ }^{2}$ and Beatriz Martínez ${ }^{1 *}$
}

${ }^{1}$ DairySafe Group, Instituto de Productos Lácteos de Asturias (IPLA)-Consejo Superior de Investigaciones Científicas (CSIC), Villaviciosa, Spain, ${ }^{2}$ Chr. Hansen A/S, Hørsholm, Denmark

Lactococcus lactis is widely used as a starter in the manufacture of cheese and fermented milk. Its main role is the production of lactic acid, but also contributes to the sensory attributes of cheese. Unfortunately, the diversity of suitable strains to be commercialized as dairy starters is limited. In this work, we have applied adaptive evolution under cell envelope stress (AE-CES) as means to provide evolved L. lactis strains with distinct physiological and metabolic traits. A total of seven strains, three of industrial origin and four wild nisin Z-producing L. lactis, were exposed to subinhibitory concentrations of Lcn972, a bacteriocin that triggers the cell envelope stress response in L. lactis. Stable Lcn972 resistant (Lcn972R) mutants were obtained from all of them and two mutants per strain were further characterized. Minimal inhibitory Lcn972 concentrations increased from 4- to 32-fold compared to their parental strains and the Lcn972R mutants retained similar growth parameters in broth. All the mutants acidified milk to a $\mathrm{pH}$ below 5.3 with the exception of one that lost the lactose plasmid during adaptation and was unable to grow in milk, and two others with slower acidification rates in milk. While in general phage susceptibility was unaltered, six mutants derived from three nisin Z producers became more sensitive to phage attack. Loss of a putative plasmid-encoded anti-phage mechanism appeared to be the reason for phage susceptibility. Otherwise, nisin production in milk was not compromised. Different inter- and intra-strain-dependent phenotypes were observed encompassing changes in cell surface hydrophobicity and in their autolytic profile with Lcn972R mutants being, generally, less autolytic. Resistance to other antimicrobials revealed cross-protection mainly to cell wall-active antimicrobials such as lysozyme, bacitracin, and vancomycin. Finally, distinct and shared non-synonymous mutations were detected in the draft genome of the Lcn972R mutants. Depending on the parental strain, mutations were found in genes involved in stress response, detoxification modules, cell envelope biogenesis and/or nucleotide metabolism. As a whole, the results emphasize the different strategies by which each strain becomes resistant to Lcn972 and supports the feasibility of AE-CES as a novel platform to introduce diversity within industrial L. lactis dairy starters.

Keywords: dairy starter, bacteriocin, stress, adaptive evolution, cell wall 


\section{INTRODUCTION}

Dairy starters have been applied for the production of fermented dairy products more than a century ago, when the first dairy starter strains were isolated and intentionally added to milk. The main components of dairy starters are lactic acid bacteria (LAB) and, in particular, Lactococcus lactis is the most common acidifying strain used in the production of cheese. According to their composition, cheese starters are classified into undefined and defined starters. Undefined starters are complex mixtures of unknown composition, whereas defined starters are blends of well-characterized strains of one (single) or multiple (mixed) species (Rodríguez et al., 2012). These starter strains have been isolated and selected according to their technological properties, namely based on fast growth and acidification rate in milk, proteolytic activity and bacteriophage resistance. Other features such as the synthesis of aroma compounds, texturing agents and inhibitory compounds are also of interest (Derkx et al., 2014). Commercial defined starters are currently available in highly concentrated frozen and freeze-dried formats, ready to be added directly to vat milk to minimize the risk of starter contamination during handling and changes in starter composition in the factory. Overall, the use of starters results in reliable cheese quality and, most importantly, in a more consistent acidification rate that allows cheese making be conducted on a fixed time schedule (Johnson, 2017).

Changes in consumer preferences toward less additives and artificial ingredients in fermented products put pressure on companies engaged in the production of starters to expand their strain portfolio to satisfy these demands (Johansen et al., 2015). However, there is a general consensus that the biodiversity of available commercial starters is relatively small to develop defined starter blends for novel applications. Consequently, either new sources or novel strategies for strain development are required (McAuliffe, 2018). Starter improvement may be approached by knowledge-based screenings of large culture collections, looking for the desired combination of properties, or by evolving new phenotypes from existing starters. The former approach is limited by the fact that not all strains survive the conditions imposed by industrial production, whereas the latter may be applied to strains whose production at large-scale has been already optimized. The success of strain improvement based on natural selection and experimental evolution has been recently reviewed by Johansen (2018). Besides classical mutagenesis and standard or cuttingedge genome editing technologies, knowledge-based strategies to diversify may include positive selection under a particular condition. For example, to increase yogurt sweetness, growth of Streptococcus thermophilus mutants able to consume the galactose moiety of lactose and excrete the glucose moiety could be isolated. First, growth on galactose to select for galactose positive mutants was approached, followed by subsequent selection on the non-metabolized glucose analog 2-deoxyglucose (Sørensen et al., 2016). Additionally, adaptive evolution experiments that involved serial propagation under selective conditions have also been applied to LAB to evolve strains with specific phenotypes (see Bachmann et al., 2017 for a recent review). Examples of adaptive evolution in L. lactis include adaptation to high temperature (Chen et al., 2015), or adaptation of a plant isolate to grow in milk (Bachmann et al., 2012).

An alternative route to diversify $L$. lactis populations may be the use of bacteriocins in evolution experiments. Bacteriocins are bacterial ribosomally synthesized antimicrobial peptides. Those produced by LAB have received much attention due to their biotechnological potential as food biopreservatives, based on their potent inhibitory activity against foodborne pathogens and spoilage microorganisms. LAB bacteriocins encompass a large and diverse group of peptides with multiple structures that kill target bacteria by interfering with cell envelope functions, either by disruption of membrane permeability by pore formation and/or inhibition of cell wall synthesis (Álvarez-Sieiro et al., 2016). It has also been shown that bacteriocin resistant mutants (BacR) can be isolated under laboratory conditions. BacR mutants may display altered phenotypes from changes in cell wall constituents and membrane fluidity to alterations of carbon metabolism. The latter occurs namely by mutation or repression of bacteriocin receptors which are also involved in sugar uptake (Tessema et al., 2011; Bastos et al., 2015). However, most of these studies have focused on mutants of main foodborne pathogens such as Listeria monocytogenes or Staphylococcus aureus. Much less is known on the impact of bacteriocin resistance in industrially relevant bacteria such as L. lactis. Nisin resistance in L. lactis IL1403 has been linked to an increased D-alanylation of lipoteichoic acids and a thickened cell wall at the septum (Kramer et al., 2008). Transcriptomic studies further suggested that other mechanisms (e.g., ABC transporters and metabolic adaptations) may be also involved (Kramer et al., 2006). The phenotypic consequences of resistance to the lantibiotic lacticin 3147 have also been characterized in L. lactis IL1403 (Guinane et al., 2006). Resistance to other anti-lactococcal bacteriocins such as lactococcin $\mathrm{G}$ and LsbB has been associated with mutations of the enzyme undecaprenyl pyrophosphate phosphatase (UppP), involved in cell wall biosynthesis, and of a membrane metallopeptidase (YvjB), respectively (Kjos et al., 2014; Miljkovic et al., 2016). These proteins are suggested to be bacteriocin receptors required for antimicrobial activity.

Lactococcin 972 (Lcn972) is a non-pore forming bacteriocin only active against Lactococcus that inhibits cell wall biosynthesis at the septum by specifically binding to lipid II (Martínez et al., 2008). In line with this mode of action, Lcn972 triggers the cell envelope stress response in Lactococcus through the activation of the two component system CesSR (Martínez et al., 2007). Although we were not able to isolate spontaneous mutants after a single exposure to Lcn972, a stable resistant mutant of the laboratory strain L. lactis MG1614 could be selected by subculturing in the presence of increasing Lcn 972 concentrations. The characterization of a single resistant mutant demonstrated that changes on the cell surface, along with chromosomal deletions and transcriptional gene activation mediated by insertion sequences, had occurred (Roces et al., 2012a,b). This mutant revealed cross-resistance to lysozyme and nisin and insensitivity to the bacteriophage c2 (Roces et al., 2012a). More recently, to assess if industrial strains are also prone to Lcn972 plasticity, we have applied the same procedure to the cheese starter L. lactis IPLA947. L. lactis R5, a Lcn972 resistant 
mutant (Lcn972R) four times more resistant than L. lactis IPLA947, showed increased resistance to oxidative stress without compromising the acidification rate in milk (López-González et al., 2018).

These previous results encourage us to propose that Adaptive Evolution under Cell Envelope Stress (AE-CES), using Lcn972 as a stressor, might be an option to diversify industrial L. lactis, i.e., to introduce new phenotypes in existing dairy starters. Thus, the aim of this work was to apply AE-CES to seven L. lactis strains of different origins (industrial and raw milk cheese isolates), and assess the phenotypes of their Lcn972R mutants with special emphasis in milk growth, surface properties and stress survival. Both unique and common phenotypes were observed. Moreover, distinct point mutations could be detected in the draft genome sequence of thirteen Lcn972R mutants that revealed the heterogeneity of the different strategies of L. lactis to cope with the stress imposed by Lcn972.

\section{MATERIALS AND METHODS}

\section{Bacterial Strains and Growth Conditions}

Lactococcus lactis strains used in this work and their sources and properties are shown in Table 1. Additionally, L. lactis IPLA947 (Cárcoba et al., 2000), the acidifying strain of the Afuega'l Pitu cheese starter, and its Lcn972R mutant R5 (López-González et al., 2018) were also included for the phenotypic and genotypic tests. L. lactis was routinely grown at $30^{\circ} \mathrm{C}$ statically in LM17 (Biokar Diagnostics) that incorporates lactose at $0.5 \%(\mathrm{w} / \mathrm{v})$ in its composition. For testing lactose fermentation, a basal broth was prepared with: (w/v) $0.5 \%$ tryptone (Oxoid), $0.3 \%$ meat extract (Biokar Diagnostics), and bromocresol purple dye (BCP) at $0.004 \%$ supplemented with either lactose (BCP-lac) or glucose (BCP-glc) at 0.5\%. L. lactis MG1614 was grown in M17 (Oxoid) supplemented with glucose (GM17) at $0.5 \%$ and used as indicator for Lcn972 quantification. Micrococcus luteus NCIMB8166 was used as indicator for nisin quantification and was grown in Tryptic Soy broth (TSB, Biokar Diagnostics) at $37^{\circ} \mathrm{C}$. Plates were prepared with agar at $2 \%$. Frozen stocks were kept at $-80^{\circ} \mathrm{C}$ in the presence of $10 \%$ glycerol. Working culture stocks were prepared from overnight (16-18 h) cultures started with a single colony. Glycerol was added to a final concentration of $10 \%$ and $40 \mu \mathrm{l}$-aliquots were stored at $-80^{\circ} \mathrm{C}$. Before each experiment, one aliquot was unfrozen and used to inoculate $4 \mathrm{ml}$ of LM17. Growth curves were carried out in a Benchmark Plus Microplate spectrophotometer (Bio-Rad Laboratories). Growth was started by diluting overnight cultures in pre-warmed LM17 to an optical density at $600 \mathrm{~nm}\left(\mathrm{OD}_{600}\right)$ of 0.05 . Growth rate $(\mu)$ was calculated by linear regression of $\ln \left(\mathrm{OD}_{600}\right)$ versus time in the exponential phase in, at least, two independent experiments.

\section{Minimal Inhibitory Concentration}

Susceptibility to Lcn972 was determined by the broth microdilution method as previously described (Martínez et al., 2008). Purified Lcn972 stock was prepared in $50 \mathrm{mM}$ sodium phosphate buffer, $\mathrm{pH}$ 6.8, with a specific activity of

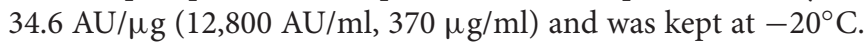

Twofold dilutions $(100 \mu \mathrm{l})$ were made in broth and placed on microtiter plates. Wells were inoculated with $100 \mu \mathrm{l}$ of an exponentially growing culture adjusted to $\mathrm{OD}_{600}$ of 0.05 and further diluted 1:100. Plates were incubated for $24 \mathrm{~h}$ at $30^{\circ} \mathrm{C}$.

\section{Adaptive Evolution Experiments}

Adaptation to Lcn972 proceeded essentially as described by Roces et al. (2012a) and is depicted in Figure 1A. Cultures were started with a single colony in LM17. After overnight incubation at $30^{\circ} \mathrm{C}$, they were used to inoculate at $1 \%(\mathrm{v} / \mathrm{v}) 10 \mathrm{ml}$ of prewarmed LM17 containing $10 \mathrm{AU} / \mathrm{ml}$ of Lcn972. After incubation at $30^{\circ} \mathrm{C}$ for $16 \mathrm{~h}$, the same procedure was applied, doubling the concentration of $\operatorname{Lcn} 972$ in each round. A total of eight consecutive transfers were done into fresh LM17, starting at $10 \mathrm{AU} / \mathrm{ml}$ up to $1,280 \mathrm{AU} / \mathrm{ml}$, with the exception of L98 that was stopped after 5 transfers at $160 \mathrm{AU} / \mathrm{ml}$. Decimal dilutions of each culture grown at the highest Lcn972 concentration were spread on LM17 plates without Lcn972 to isolate single colonies. From L. lactis L81 and L62, 43, and 30 colonies were picked, respectively. For nisin producers, 16 colonies per strain were picked (Figure 1B). Each colony was transferred to 1-ml deepwell microtiter plates filled with LM17 and grew for $24 \mathrm{~h}$ at $30^{\circ} \mathrm{C}$. These microtiter plates were replicated by inoculating at $1 \%(\mathrm{v} / \mathrm{v})$ new LM17 deepwell plates that were incubated at $30^{\circ} \mathrm{C}$ for $24 \mathrm{~h}$. After 10 serial transfers, the susceptibility to Lcn972 was checked. To do so, cells from each well were diluted 1:100 in the case of L. lactis L81 and L62 or 1:160 for nisin producers in fresh LM17 and $5 \mu \mathrm{l}$ were used to inoculate $200 \mu \mathrm{l}$ of LM17 plus Lcn972 at $320 \mathrm{AU} / \mathrm{ml}$ (L81 and L62) or $80 \mathrm{AU} / \mathrm{ml}$ (nisin producers). Two cultures per L. lactis strain able to grow in the presence of Lcn972 were streaked on a LM17 plate and a single colony was stored at $-80^{\circ} \mathrm{C}$.

\section{Milk Acidification and Production of Lactic Acid}

Skim milk powder (Difco) was reconstituted at 11\% (w/v) with distilled water and treated at $100^{\circ} \mathrm{C}$ for $30 \mathrm{~min}$. Overnight LM17 cultures were centrifuged and washed once with Ringer solution (Merck, Germany). Milk (10 ml) was inoculated at 3\% (v/v) with the cell suspension and incubated at $30^{\circ} \mathrm{C}$ for $6 \mathrm{~h} . \mathrm{pH}$ was measured with the pHmeter micropH 2001 (Crison, Spain). Acidification curves of L81 and L62 and their Lcn972 resistant mutants (Lcn972R) were started by inoculation of $40 \mathrm{ml}$ of milk with the cell suspension at $2 \%(\mathrm{v} / \mathrm{v})$ and $\mathrm{pH}$ was followed during the incubation at $30^{\circ} \mathrm{C}$ with the real-time pHmeter Orion ${ }^{\mathrm{TM}}$ Versa $^{\mathrm{TM}}$ Start (Thermo Scientific Inc., United States) every $30 \mathrm{~min}$ for $20 \mathrm{~h}$. Maximum acidification rate $\left(V_{\mathrm{m}}\right)$ and the time at which $\mathrm{pH} 4.6$ was reached (Te) were used as acidification parameters as described by Kristo et al. (2003).

Lactic acid production was analyzed by HPLC using an ICSepICE-ION-300 column with $0.0085 \mathrm{~N} \mathrm{H}_{2} \mathrm{SO}_{4}$ as mobile phase at flow rate of $0.4 \mathrm{ml} / \mathrm{min}$ and operating temperature of $65^{\circ} \mathrm{C}$. Lactic acid was detected at $210 \mathrm{~nm}$ by a 996 photodiode array detector (Waters). Milk samples $(1 \mathrm{ml})$ were deproteinized with $5 \mathrm{ml}$ of $4.5 \mathrm{mM} \mathrm{H}_{2} \mathrm{SO}_{4}$ for $1 \mathrm{~h}$ at $37^{\circ} \mathrm{C}$ in a rotary shaker. After centrifugation $\left(16,000 \times g, 15 \mathrm{~min}, 4^{\circ} \mathrm{C}\right)$, supernatants were 
TABLE 1 | Source and properties of Lactococcus lactis subsp. lactis strains used in this work.

\begin{tabular}{|c|c|c|c|c|}
\hline Strain-code & Source and properties & Reference & Lcn972 MIC ${ }^{1}$ (AU/ml) & Growth rate ${ }^{2}\left(\mathbf{h}^{-1}\right)$ \\
\hline L. lactis L81 & Commercial mesophilic starter. Acidifying strain & $\mathrm{CHCC}$ collection & 10 & $0.69 \pm 0.10$ \\
\hline L81-D1 & Resistant to Lcn972 & This work & 80 & $0.83 \pm 0.04^{*}$ \\
\hline L81-E2 & Resistant to Lcn972 & This work & 320 & $0.79 \pm 0.01$ \\
\hline L. lactis L62 & Commercial mesophilic starter. Acidifying strain & CHCC collection & 10 & $0.84 \pm 0.01$ \\
\hline L62-C9 & Resistant to Lcn972 & This work & 320 & $0.89 \pm 0.04$ \\
\hline L62-G9 & Resistant to Lcn972 & This work & 80 & $0.80 \pm 0.02$ \\
\hline L. lactis L98 & Bioprotective culture. Nisin A producer & $\mathrm{CHCC}$ collection & 40 & $0.95 \pm 0.01$ \\
\hline L98-C1 & Resistant to Lcn972 & This work & 160 & $0.90 \pm 0.04^{*}$ \\
\hline L98-E2 & Resistant to Lcn972 & This work & 160 & $0.81 \pm 0.09^{*}$ \\
\hline L. lactis IPLA517 & Raw milk cheese, nisin Z producer & Martínez et al., 1995 & 10 & $0.95 \pm 0.01$ \\
\hline $517-\mathrm{B} 5$ & Resistant to Lcn972 & This work & 320 & $0.74 \pm 0.06$ \\
\hline $517-\mathrm{C} 6$ & Resistant to Lcn972 & This work & 160 & $0.82 \pm 0.16$ \\
\hline L. lactis IPLA641 & Raw milk cheese, nisin Z producer & Martínez et al., 1995 & 10 & $0.98 \pm 0.09$ \\
\hline $641-\mathrm{C} 8$ & Resistant to Lcn972 & This work & 80 & $0.87 \pm 0.09$ \\
\hline 641-D8 & Resistant to Lcn972 & This work & 160 & $0.79 \pm 0.01$ \\
\hline L. lactis IPLA729 & Raw milk cheese, nisin Z producer & Martínez et al., 1995 & 10 & $1.08 \pm 0.04$ \\
\hline 729-D10 & Resistant to Lcn972 & This work & 160 & $0.97 \pm 0.00$ \\
\hline $729-F 9$ & Resistant to Lcn972 & This work & 160 & $0.90 \pm 0.03^{*}$ \\
\hline L. lactis IPLA1064 & Raw milk cheese, nisin Z producer & Martínez et al., 1995 & 10 & $0.97 \pm 0.00$ \\
\hline $1064-C 11$ & Resistant to Lcn972 & This work & 160 & $0.81 \pm 0.01$ \\
\hline 1064-E11 & Resistant to Lcn972 & This work & 160 & 0.78 \\
\hline
\end{tabular}

${ }^{1}$ MIC, Minimal Inhibitory Concentration. AU, Arbitrary Units.

${ }^{2}$ Growth rate in $L M 17$ broth at $30^{\circ} \mathrm{C}$. Results are the mean \pm standard deviation $(n=2)$.

*Statistically different $(p<0.05)$ compared to the equivalent wild-type strain.

filtered through a $0.45 \mu \mathrm{m}$ PTFE filter (VWR international). All the experiments were carried out with at least two independent cultures.

\section{Nisin Production}

Nisin was quantified by the agar well diffusion method. Plates were prepared by inoculating melted TSB agar $1.2 \%$ with $10^{5}$ colony forming unit (CFU) per $\mathrm{ml}$ of $M$. luteus NCIMB8166. After solidification, wells (4 $\mathrm{mm}$ diameter) were made and filled with $20 \mu \mathrm{l}$ of culture or milk supernatants obtained by centrifugation $\left(15,400 \times g, 15 \mathrm{~min}, 4^{\circ} \mathrm{C}\right)$. Plates were incubated at $37^{\circ} \mathrm{C}$ for $24 \mathrm{~h}$ to score inhibition halos. A calibration curve was prepared with pure nisin (a gift from Applin \& Barret, United Kingdom) with concentrations from 5 to $25 \mu \mathrm{g} / \mathrm{ml}$ diluted in $0.05 \%$ acetic acid. Quantification was performed in two independent cultures.

\section{Bacteriophage Susceptibility}

Different phages from the Chr. Hansen collection infecting L. lactis L81, L62, and L98 were tested by the double layer assay. Phage decimal dilutions prepared in LM17 were spotted $(10 \mu \mathrm{l})$ onto LM17 plates containing $10 \mathrm{mM} \mathrm{Ca}^{++}$and overlaid with soft LM17 agar (0.7\%) inoculated with $100 \mu$ l of the L. lactis overnight culture. Clear halos and isolated lysis plaques were observed after overnight incubation at $30^{\circ} \mathrm{C}$. When required, plaque forming units per $\mathrm{ml}(\mathrm{PFU} / \mathrm{ml})$ were estimated after incubating $100 \mu \mathrm{l}$ of the phage dilution with $100 \mu \mathrm{l}$ of the bacterial culture for $10 \mathrm{~min}$, then mixed with $8 \mathrm{ml}$ of soft LM17 and poured on a LM17 plate. To identify phages infecting the L. lactis nisin producers IPLA517, IPLA641, IPLA729, and IPLA1064, a growth inhibition test in milk was carried out. Bacterial cultures were challenged with 62 phages at $c a .10^{6} \mathrm{PFU} / \mathrm{ml}$ and the $\mathrm{pH}$ was monitored. When the $\mathrm{pH}$ of a control culture without added phage was at 5.0 , the $\mathrm{pH}$ of the infected culture was recorded, and a phage was considered infective when the $\mathrm{pH}$ difference was more than 0.5 .

\section{Surface Properties and Autolysis}

Cell surface hydrophobicity was measured by the MicrobialAdhesion To Solvent (MATS) protocol using hexadecane and stationary phase cells as described by Roces et al. (2012a). The degree of autolysis was determined according to Meyrand et al. (2007). Briefly, exponentially growing cells $\left(\mathrm{OD}_{600}=0.3-0.5\right)$ were harvested, washed with $50 \mathrm{mM}$ potassium phosphate buffer, $\mathrm{pH} 7.0$, and resuspended in the same buffer supplemented with Triton X-100 at $0.05 \%$. The $\mathrm{OD}_{600}$ of $300 \mu \mathrm{l}$-aliquots of the cell suspension was followed in the microtiter reader for $6 \mathrm{~h}$ at $30^{\circ} \mathrm{C}$. Three biological replicates per strain were assayed. Cell suspensions without Triton X-100 were used as controls. The $\mathrm{OD}_{600}$ values were expressed as $\%$ of the initial $\mathrm{OD}_{600}$. For comparison, values of $\% \mathrm{OD}_{600}$ after $150 \mathrm{~min}$ of incubation were taken.

\section{Survival to Heat and Acidic pH}

Cells from overnight cultures were harvested by centrifugation $(15,400 \times g, 5 \mathrm{~min})$. For the heat shock, cells from $200 \mu \mathrm{l}$ aliquots were washed with Ringer solution and diluted in $1 \mathrm{ml}$ 
A

Step 1: adaptation

(+ Lcn972)

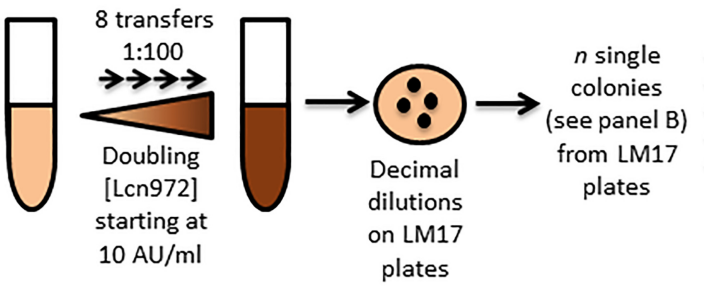

B
Step 2: stabilization

(- Lcn972)

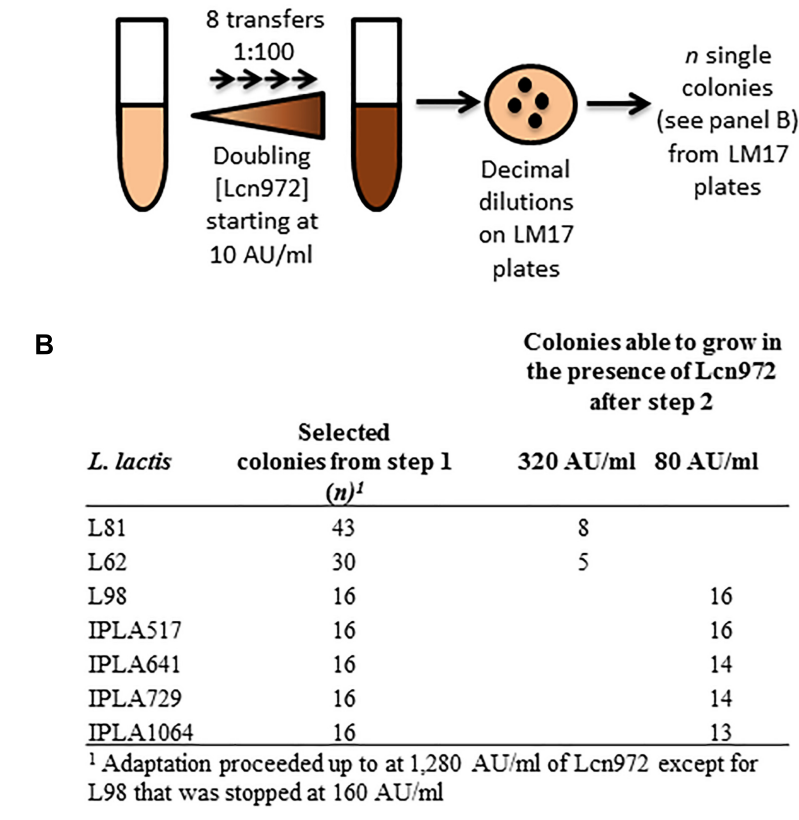

FIGURE 1 | Outline of the adaptive evolution experiment to isolate Lcn972R mutants. (A) During the adaptation step, Lactococcus lactis strains were subcultured in LM17 with increasing (twofold) Lcn972 concentrations. After this step, several colonies from the adapted cultures grown at the highest Lcn972 concentration were subcultured in the absence of Lcn972 (stabilization step). (B) Number of colonies subjected to the stabilization step and number of those which still retained resistance to Lcn972 after stabilization.

of the same solution (approximately $4-5 \times 10^{8} \mathrm{CFU} / \mathrm{ml}$ ). Cell suspensions were divided into two $0.5 \mathrm{ml}$-aliquots, one was hold in a thermoblock (VWR international) at $50^{\circ} \mathrm{C}$ for $30 \mathrm{~min}$ and the other left at room temperature. All the tubes were transferred to ice and decimal dilutions immediately prepared in Ringer solution. $\mathrm{CFU} / \mathrm{ml}$ counts were estimated after spotting $(6 \times) 5 \mu \mathrm{l}$ of each dilution on LM17 plates. The experiment was carried out with three biological replicates. For the acidic shock, cells from $1 \mathrm{ml}$ were washed twice with $150 \mathrm{mM} \mathrm{NaCl}$, and finally resuspended in $0.5 \mathrm{ml}$ of $150 \mathrm{mM} \mathrm{NaCl}$. Each cell suspension $(100 \mu \mathrm{l})$ was diluted in $900 \mu \mathrm{l}$ of $150 \mathrm{mM} \mathrm{NaCl}$ and in $150 \mathrm{mM}$ $\mathrm{NaCl}$ adjusted to $\mathrm{pH} 2$ with $\mathrm{HCl}$. Samples were prepared in duplicate and incubated for $30 \mathrm{~min}$ at $30^{\circ} \mathrm{C}$. After the incubation, the tubes were centrifuged and the pellet resuspended in $1 \mathrm{ml}$ of phosphate buffered saline (PBS, $10 \mathrm{mM} \mathrm{Na} \mathrm{HPO}_{4}, 1.8 \mathrm{mM}$ $\mathrm{KH}_{2} \mathrm{PO}_{4}, 137 \mathrm{mM} \mathrm{NaCl}, 2.7 \mathrm{mM} \mathrm{KCl}, \mathrm{pH}$ 7.4). Decimal dilutions were prepared in PBS to neutralize the $\mathrm{pH}$ and $\mathrm{CFU} / \mathrm{ml}$ calculated as described after the heat shock. Survival was determined as $\log (\mathrm{Nt} / \mathrm{No})$ where $\mathrm{Nt}$ and $\mathrm{No}$ are $\mathrm{CFU} / \mathrm{ml}$ of treated samples and non-treated controls, respectively. In both experiments, three independent cultures per strain were used.

\section{Resistance to Antimicrobials}

Decimal dilutions of exponentially growing cultures $\left(\mathrm{OD}_{600}=0.3-0.5\right)$ were prepared in Ringer solution and spotted $(5 \mu \mathrm{l})$ on LM17 plates supplemented with each antimicrobial at final concentrations of $1 \mu \mathrm{g} / \mathrm{ml}$ bacitracin, $0.1 \mathrm{mg} / \mathrm{ml}$ penicillin $\mathrm{G}, 0.4 \mu \mathrm{g} / \mathrm{ml}$ vancomycin and $0.5 \mathrm{mg} / \mathrm{ml}$ lysozyme from chicken egg white (all purchased from Sigma). LM17 plates supplemented with $5 \% \mathrm{NaCl}$ were also prepared. Resistance to nisin $(5 \mu \mathrm{g} / \mathrm{ml})$ was also checked for all the starter strains and Lcn972R mutants but the nisin producers.

\section{Plasmid Isolation and Plasmid Curation}

Plasmids were isolated from overnight cultures according to O'sullivan and Klaenhammer (1993). For curing experiments, L. lactis IPLA517 was grown in LM17 at $37^{\circ} \mathrm{C}$ for $24 \mathrm{~h}$ in the presence of novobiocin at $2 \mu \mathrm{g} / \mathrm{ml}$. These cultures were transferred daily to LM17 with increasing novobiocin concentrations $(5,10$, and $15 \mu \mathrm{g} / \mathrm{ml})$. Cultures grown at $15 \mu \mathrm{g} / \mathrm{ml}$ of novobiocin were spread on LM17 plates to isolate single colonies.

\section{Identification of Non-synonymous Single Point Mutations}

Draft genomes sequences were obtained at the sequencing facilities at Chr. Hansen. To perform full genome sequencing, DNA of the selected strains was isolated using the DNA DNeasy Blood and Tissue kit with the protocol for Gram-positive bacteria (Qiagen, Germany) and sequenced on the Illumina MiSeq platform with $2 \times 250$-bp paired-end sequencing (Illumina, United States). Sequencing reads were trimmed, analyzed, and 
assembled using CLC Genomics Workbench 10.1.1 (Invitrogen). The assembled contigs were annotated by RASTtk (Brettin et al., 2015). Whole Genome Shotgun projects have been deposited at DDBJ/ENA/GenBank under the bioproject accession number PRJNA492214. Detection of single point mutations in the genomes of the Lcn972R mutants was performed with CLC Genomics Workbench 10.1.1 (Invitrogen). Variants located in mobile elements and prophages were disregarded. Moreover, BLASTN $^{1}$ was used to identify possible sequencing/assembling errors found in the wild type genome used as reference due to low coverage or low quality readings. Mutations found in plasmidencoded genes which were also present in the chromosome (e.g., oligopeptide transport genes-opp) were not included in the analysis as the extra-copy may influence the outcome of the variant caller. Heatmap for representing the mutations found in the Lcn972R mutants was prepared with Heatmapper (Babicki et al., 2016). Hierarchical clustering was done by the average linkage method and distances were computed by the Spearman rank correlation. Functional analysis of the non-synonymous mutations detected in the Lcn972R strains was performed with GSEA_pro ${ }^{2}$ as implemented in Genome2D ${ }^{3}$. The SIFT algorithm was used to predict if an amino acid substitution affects protein function ${ }^{4}$.

\section{Statistical Analysis}

Results are reported as mean \pm SD where appropriate. Differences between the wild type strains and their Lcn972R derivatives were assessed by one-tailed $t$-test as implemented in Microsoft Excel 2010 (2010 Microsoft Corporation). $p<0.05$ was considered to be significant.

\section{RESULTS}

\section{Adaptive Evolution Under Cell Envelope Stress (AE-CES) Applied to Industrial and Dairy Strains Selects for Stable $L$. lactis Mutants Resistant to Len972}

To appreciate the value of AE-CES as means to evolve L. lactis and provide new phenotypes, this procedure was applied to seven wild type (WT) L. lactis subsp. lactis strains from different sources (Table 1). L. lactis L81 and L62 are used as acidifying strains in commercial starter blends. L98 is a nisin A producer, available as a bioprotective culture. The four nisin $\mathrm{Z}$ producers (IPLA517, IPLA641, IPLA729, and IPLA1064) were selected from the IPLA-CSIC laboratory collection. These strains were isolated from homemade raw milk cheeses made without starters in Northern Spain, and were chosen as representatives of each of the plasmid profiles found among the 23 isolates characterized by Martínez et al. (1995). They are genetically very closely related, according to their identical SmaI band pattern resolved

\footnotetext{
${ }^{1}$ https://blast.ncbi.nlm.nih.gov/

${ }^{2}$ http://gseapro.molgenrug.nl/

${ }^{3}$ http://genome2d.molgenrug.nl/

${ }^{4}$ http://sift.bii.a-star.edu.sg/
}

by pulsed-field gel electrophoresis PFGE (our own unpublished results). Nonetheless, they were all subjected to AE-CES to determine if they generate similar outcomes.

The seven WT L. lactis strains were susceptible to Lcn972 with a MIC of $10 \mathrm{AU} / \mathrm{ml}$ and $40 \mathrm{AU} / \mathrm{ml}$ for L98 (Table 1). The AE-CES experiment involved two steps (see Figure 1A). In the first step, cultures were adapted to grow in increasing Lcn972 concentrations (adaptation step). All the cultures were able to grow up to $1,280 \mathrm{AU} / \mathrm{ml}(128 \times \mathrm{MIC})$ of Lcn972. The only exception was L98 that grew poorly at concentrations over $160 \mathrm{AU} / \mathrm{ml}$ (4× MIC). From each adapted culture, single colonies were picked and subjected to the stabilization step, consisting in ten serial transfers in LM17 without Lcn972. This step was carried out to enrich for the fittest clones. Then, their ability to grow in the presence of Lcn972 was tested Not all of them were able to grow (Figure 1B), suggesting that, in some cases, resistance to Lcn972 may be lost during the stabilization step. From two independent stabilized cultures per strain that retained the Lcn972R phenotype, a single colony was isolated, named after its parental strain, and stored for further characterization. Their Lcn972 MICs are shown in Table 1. MICs were increased from 4- to 32 -fold. Most frequently, the MIC value was $160 \mathrm{AU} / \mathrm{ml}$, while three mutants reached $320 \mathrm{AU} / \mathrm{ml}$. It is important to note that the inoculum for the MIC determination differs from that used in the Lcn972 test. MIC plates were inoculated with approximately $1.5 \times 10^{5} \mathrm{CFU} / \mathrm{ml}$ from exponentially growing cultures, while the Lcn972 test was carried out with stationary phase cultures and inoculated with $3.0-6.0 \times 10^{5} \mathrm{CFU} / \mathrm{ml}$. This difference in the inoculum may explain why Lcn972R mutants from L. lactis L81 and L62 had MICs below $320 \mathrm{AU} / \mathrm{ml}$, the Lcn972 concentration used in the Lcn972 test. Moreover, the MICs were also below 1,280 AU/ml, the highest concentration used during adaptation. In this case, the transitory CesSR response must be activated during adaptation, which likely helps to cope with the stress and may allow growth at high Lcn972 concentrations.

In general, according to their growth rate in LM17 (Table 1), growth of the Lcn972R mutants was not hampered under laboratory conditions. L. lactis L81-D1 grew even faster than its parent (L81) $(p<0.05)$. On the contrary, L. lactis IPLA729F9 and the two mutants from L98 were slower (Table 1). Furthermore, while the final $\mathrm{OD}_{600}$ in LM17 overnight cultures was similar to that of the parental strains (2.5-3.0) (data not shown), the mutants L98-C1 and L98-E2 reached a low $\mathrm{OD}_{600}$ compared to that of L98 (0.8 vs. 2.5 , respectively). The bioprotective culture L. lactis L98 had a particular behavior. When streaked on LM17 plates, two colony phenotypes were always observed with an estimated frequency of 50\%: a typical lactococcal white and smooth colony and a translucent and flat colony, which was later confirmed as lactose negative variants (Supplementary Figure S1). Adaptation was started with a lactose positive colony but it seems that during this step, only lactose negative variants were selected. In fact, lack of lactose fermentation and loss of a large plasmid band in L98-C1 and L98-E2 was confirmed by the absence of growth in BCP-lac (Supplementary Figure S2C) and plasmid isolation, respectively (Supplementary Figure S2A). Another mutant that lost one 

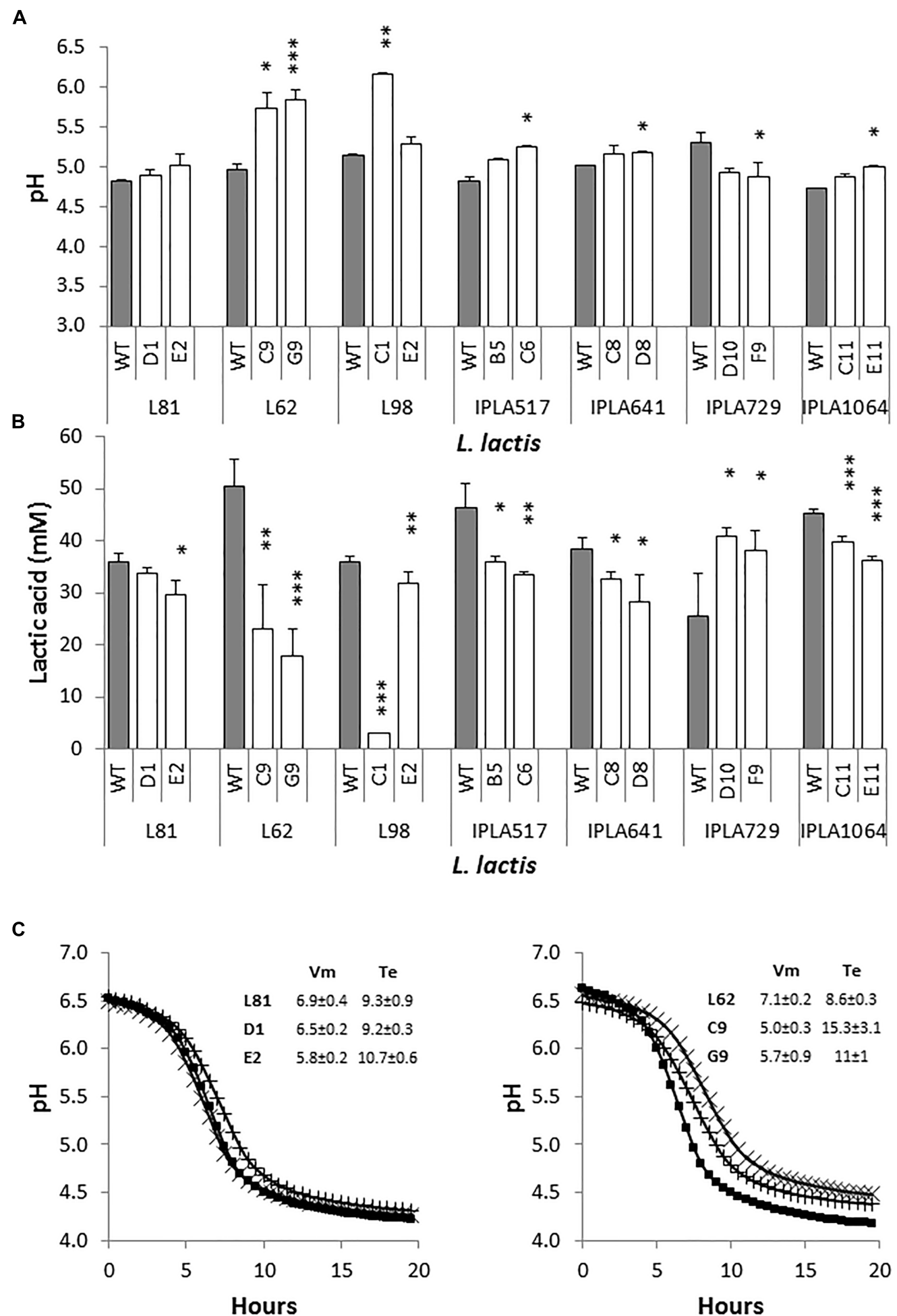

FIGURE 2 | Growth of L. lactis in pasteurized milk. $\mathrm{pH}$ (A) and production of lactic acid (B) of L. lactis wild-type (gray bars) and their Lcn972R mutants (white bars). Cultures were inoculated at $3 \%$ and incubated for $6 \mathrm{~h}$ at $30^{\circ} \mathrm{C}$. (C) Acidification curves of L. lactis L81 (left panel) and L. lactis L62 (right panel) and their Lcn972R mutants in pasteurized milk. (口), wild type strains; (X), L81-D1 and L62-C9; (+), L81-E2 and L62-G9. The insert shows the acidification curve descriptors. Vm, maximum acidification rate $(\mathrm{dpH} / \mathrm{dt}, \mathrm{mpH} / \mathrm{min}) ;$ Te, time to reach $\mathrm{pH} 4.6$ (h). ${ }^{*} p<0.05,{ }^{* *} p<0.01,{ }^{* * *} p<0.001$ significantly different to the wild type strain. 
plasmid was L62-C9 (Supplementary Figure S2) but in this case, lactose fermentation was not impaired (data not shown).

\section{Technological Aptitude of Len972R Mutants in Milk}

The first step in the characterization of the Lcn972R mutants selected after AE-CES was to assess whether dairy technological traits were retained or not. Accordingly, their ability to grow in pasteurized milk was compared to that of their WT counterparts. Similarly, nisin production was examined as well as bacteriophage resistance.

\section{Growth and Lactic Acid Production in Milk}

Starting with overnight cultures in LM17, pasteurized milk was inoculated at $3 \%(\mathrm{v} / \mathrm{v})$ and $\mathrm{pH}$ as well as the production of lactic acid of milk cultures was measured after $6 \mathrm{~h}$ of incubation at $30^{\circ} \mathrm{C}$ (Figure 2). All strains acidified to a $\mathrm{pH}$ below a threshold of 5.3, regarded as a standard for a dairy starter under these experimental conditions (Cogan et al., 1997). The exception was the Lcn972R mutants of the starter L. lactis L62 and the lactose negative L. lactis L98-C1 which hardly acidified milk. In addition, the mutant L98-E2, that was shown to be also lactose negative in broth, lowered the $\mathrm{pH}$ as much as the WT L. lactis L98 $(p>0.05)$ (Figure 2A). This behavior may reflect the ability of this mutant to use other energy sources from milk.

Differences were observed in the production of lactic acid after $6 \mathrm{~h}$ of incubation (Figure 2B). In general, the amount of lactic acid generated by the Lcn972R mutants ranged from 70 to $90 \%$ of that produced by their parental strains with the exception of L. lactis IPLA729-D10 and F9 with higher levels compared to the WT IPLA729. L. lactis L62-C9 and L62-G9 produced less than $50 \%$ of the lactic acid levels of their WT strain. These results suggested a long lag phase in milk when these mutants were transferred from LM17 broth to milk and/or a slower acidification rate. To get a better picture of the acidification behavior of these mutants in milk, acidification was followed measuring the $\mathrm{pH}$ with a real-time $\mathrm{pH}$ meter for $20 \mathrm{~h}$. As a reference, acidification curves for L. lactis L81 and its Lcn972R mutants were also performed (Figure 2C). In agreement with the $\mathrm{pH}$ and lactic acid concentration determined after $6 \mathrm{~h}$ of incubation (Figures 2A,B), L. lactis L81-D1 behaved as its WT, while L81-E2 was slightly slower $(p<0.05)$ in terms of maximum acidification rate (Vm) (Figure 2C). Similarly, the mutants L62C9 and L62-G9 showed a slower maximum acidification rate $(p<0.05)$ and it took 7 and $2.5 \mathrm{~h}$ longer (Te), respectively, to reach $\mathrm{pH} 4.6$ compared to L. lactis L62 (Figure 2C). Therefore, these two Lcn972R mutants had difficulties to grow in milk, were slower in lowering the $\mathrm{pH}$ and were not able to reach the same $\mathrm{pH}$ as L. lactis L62.

\section{Nisin Production}

All the nisin producers were able to synthesize nisin in LM17 broth (data not shown), but the production of nisin in milk was investigated as a key technological trait which should be retained by the evolved strains. As shown in Figure 3, no significant differences $(p>0.05)$ were observed with the Lcn972R mutants derived from the nisin $\mathrm{Z}$ producing strains isolated from raw milk cheeses. As expected, no detectable levels of nisin were measured in L98-C1 milk cultures, due to the absence of growth. On the contrary, the mutant L98-E2 did produce nisin, despite its lactose negative phenotype, although levels were slightly lower $(p<0.05)$ than the WT L98 (Figure 3).

\section{Bacteriophage Resistance}

Another technological trait of interest is bacteriophage resistance since bacteriophages are one of the leading causes of fermentation failures worldwide (Garneau and Moineau, 2011). Thus, the susceptibility of the Lcn972R mutants to phages infecting the WT strains was examined. Phages infecting the industrial strains L81, L62, and L98 still propagate and reach similar phage titers when plated on the Lcn972R mutants (data not shown). Since the phage susceptibility profile of the nisin $\mathrm{Z}$ producers was unknown, a growth inhibition assay was initially carried out to select phages able to infect the WT strains. L. lactis IPLA517, IPLA641, IPLA729, and IPLA1064 were screened against 62 lactococcal phages. Five phages, all belonging to the $c 2$ family, strongly impaired acidification of all strains. Additionally, L. lactis IPLA729 was also inhibited by two extra c2 phages and L. lactis IPLA641 was partially inhibited by a 936 family phage and by an unclassified one (Supplementary Table S1). Two c2 phages, CHPC1130 and CHPC1183, were chosen to study further their infectivity on the Lcn972R mutants. Despite the effect observed in the growth inhibition assay, none or just a few lysis plaques were detected when plating undiluted lysates of CHPC1130 $\left(1.5 \times 10^{10} \mathrm{PFU} / \mathrm{ml}\right)$ and CHPC1183 $\left(4.6 \times 10^{9} \mathrm{PFU} / \mathrm{ml}\right)$ onto the WT strains IPLA517, IPLA641, and IPLA1064 (Figure 4). On the contrary, the phages did plaque on their Lcn972R mutants and lysis plaques were observed with titers up to $10^{7} \mathrm{PFU} / \mathrm{ml}$ (Figure 4). No changes in phage susceptibility were detected for L. lactis IPLA729 and its Lcn972R mutants.

We attempted to find a plausible explanation for the phage infectivity in the Lcn972R mutants. Since plasmid loss had been already observed for some Lcn972R mutants, we speculated that a putative plasmid-encoded phage resistance mechanism could have been lost during adaptation. To test this hypothesis, we attempted to compare the plasmid profiles of these strains and their Lcn972R mutants but it was not possible to clearly discriminate if any of the plasmids were lost (data not shown). As an alternative, we used novobiocin as a plasmid-curing agent to cure plasmids from L. lactis IPLA517. After several passages, ten single colonies were grown and tested against the phage CHPC1130. Lysis plaques were observed in all of them with titers even higher than those found on the Lcn972R mutant 517-B5 (Figure 4C). All the cured clones seem to have lost several large plasmids present in L. lactis IPLA517 (Supplementary Figure S3). Based on these results, a likely explanation for the phage susceptibility of the Lcn972R mutants is the loss of plasmidencoded anti-phage mechanisms.

\section{Surface Hydrophobicity and Autolytic Behavior of Lcn972R Mutants}

A common theme in bacteriocin resistance is the existence of changes of the physicochemical properties of the bacterial surface 


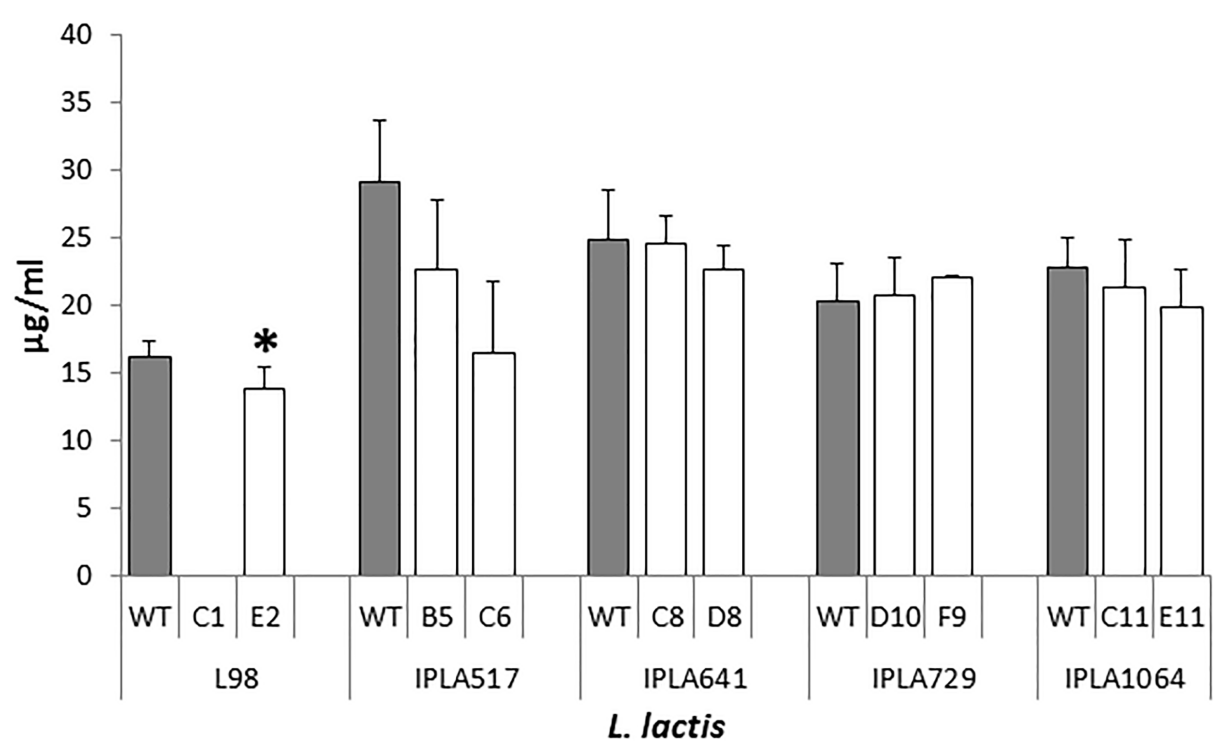

FIGURE 3 | Nisin production by L. lactis and their Lcn972R mutants (white bars) in pasteurized milk incubated at $30^{\circ} \mathrm{C}$ for $6 \mathrm{~h}$. ${ }^{*}$, significantly different $(p<0.05$ ) from the wild-type (gray bars).

to prevent or reduce binding of the antimicrobial peptide to the cell (Kramer et al., 2008; Bastos et al., 2015). Moreover, alterations on the bacterial surface may influence the activity of autolysins (Steen et al., 2005). In this context, we studied cell hydrophobicity and autolysis within the Lcn972R mutants. In these experiments, the starter L. lactis IPLA947 and its Lcn972 resistant derivative L. lactis $\mathrm{R} 5$ were also included.

Nine Lcn972R mutants changed their surface hydrophobicity with respect to the parental strains (Figure 5A). Within the group of nisin Z producers IPLA517, IPLA641, and IPLA1064 and the starter IPLA947, their Lcn972R mutants were more hydrophobic with values between 20 and $40 \%$, as measured by their transfer to the hexadecane phase in the MATS test. A dramatic shift in surface hydrophobicity was noted for L62 whose Lcn972R mutants completely lost its hydrophobic character. Mutants from L. lactis L81, L98, and IPLA729 did not suffer any major changes in surface hydrophobicity.

As to their autolytic behavior, the Lcn972R mutants became less autolytic than their parental strains (Figure 5B) with two exceptions: L. lactis L81-E2 and L. lactis R5. In both Lcn972R mutants, down to $40 \%$ of the initial $\mathrm{OD}_{600}$ was recorded after $150 \mathrm{~min}$ in the presence of triton X-100 while $60 \%$ was recorded for the WT strains. The Lcn972R mutants from L. lactis L62 behave as the WT strain (Figure 5B). It is interesting to note that the two Lcn972R mutants from the same parental strain, e.g., L. lactis L81, behaved opposite to each other with regard to their autolytic activity (Figure 5B).

\section{Cross-Resistance to Technological Stresses and Cell Wall Antimicrobials}

Considering that Lcn972 inhibits cell wall biosynthesis and triggers the cell envelope stress response in L. lactis, it was anticipated that mutations leading to changes in cell wall structure or composition could have been selected for during adaptation. Because the cell wall is crucial for survival (Chapot-Chartier and Kulakauskas, 2014), we presumed that these possible alterations could make Lcn972R mutants to withstand better other stresses such as heat, low $\mathrm{pH}$, and high osmotic pressure, all of them technological stresses encountered during cheese production. Besides, cross-resistance to other antimicrobials acting at the cell wall might arise, as previously observed with the laboratory strain L. lactis MG1614 that became resistant to lysozyme and nisin (Roces et al., 2012a). Therefore, the following experiments were designed to compare the response of the Lcn972R mutants to their parental strains.

\section{Cross-Resistance to Heat and Low pH}

A representative set of Lcn972 mutants, including those from the industrial L. lactis L81, L62, and L98, two nisin Z producers IPLA517 and IPLA641, and the dairy starter IPLA947 were exposed to $50^{\circ} \mathrm{C}$ and the viability was determined after $30 \mathrm{~min}$ (Figure 6A). Out of the eleven Lcn972R mutants, one, L. lactis $\mathrm{R} 5$, was more resistant to heat shock and three equally resistant to their wild types (L81-D1, IPLA517-B5, and IPLA517-C6). All the others became sensitized and lost around 1 log unit more than their wild types (Figure 6A). Once again, the two Lcn972R mutants from L81 and L62 behaved opposite to each other. On the contrary, none of the Lcn972R mutants became more sensitive to low $\mathrm{pH}$. For all the strains tested, exposure to $\mathrm{pH} 2$ led to a 4-4.5 log units decrease in viability (Figure 6B) and no significant differences $(p>0.05)$ were observed for any of the Lcn972R mutants and their parental strains.

\section{Cross-Resistance to $\mathrm{NaCl}$ and Cell Wall Antimicrobials}

Cross resistance was qualitatively evaluated by spotting decimal dilutions of exponentially growing cells on LM17 plates, 


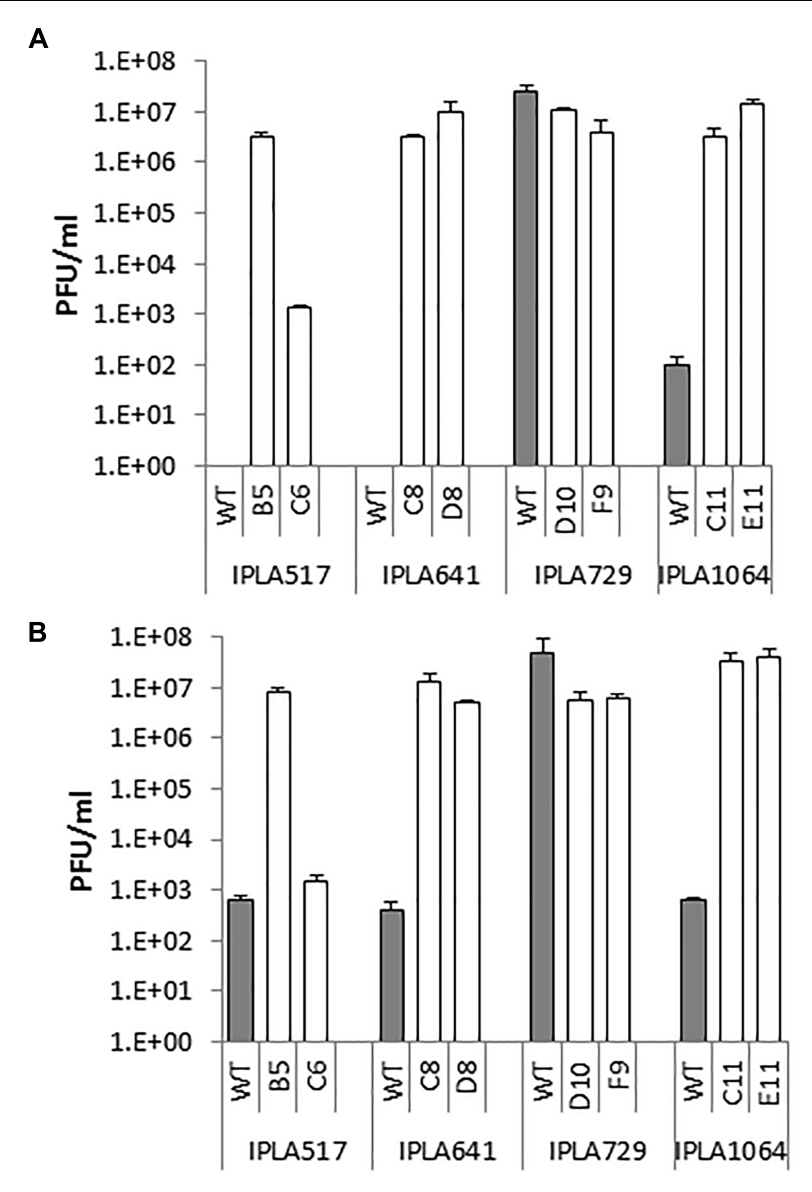

C

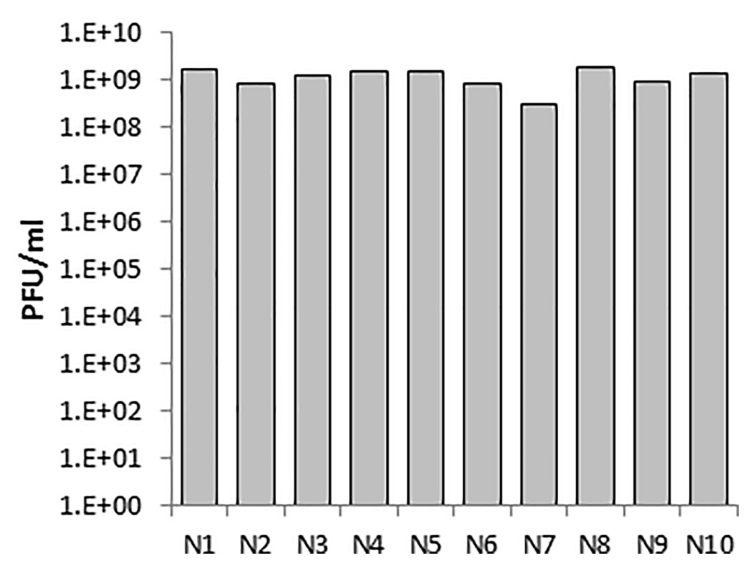

FIGURE 4 | Phage susceptibility of L. lactis IPLA517, IPLA641, IPLA729, and IPLA1064 (gray bars) and their Lcn972R mutants (white bars). Phage titer was determined by the double layer assay. (A) Phage CHPC1130 $\left(1.5 \times 10^{10}\right.$ PFU/ml). (B) Phage CHPC1180 (4.6 × $\left.10^{9} \mathrm{PFU} / \mathrm{ml}\right)$. (C) titers of phage CHPC1130 on novobiocin plasmid-cured derivatives (N1-N10) of L. lactis IPLA517.

supplemented with bacitracin, lysozyme, penicillin G and vancomycin at the concentrations indicated in Table 2. Growth under hyperosmotic conditions was assessed on LM17 plus $\mathrm{NaCl}$ at 5\% $(0.86 \mathrm{M})$. Resistance to nisin was only examined for nonnisin producers. As a general trend, half of the Lcn972R mutants became sensitive to penicillin $\mathrm{G}(60 \%)$ and to $\mathrm{NaCl}(46 \%)$. On the contrary, most of them (73\%) were more resistant to lysozyme and to vancomycin (50\%), while cross-resistance to bacitracin was observed in four cases only. Among the non-nisin producers, three out of five Lcn972R mutants showed cross-resistance to nisin. Taken as a whole, Lcn972R mutants from the same strain did not phenocopy each other and only those derived from L. lactis L98 and IPLA641 exhibited exactly the same phenotypes (Table 2).

\section{Overview of Non-synonymous Mutations Within Lcn972R Mutants}

To gain a preliminary insight into the genetic diversity introduced during evolution in the presence of Lcn972 and identify single mutations (and possible compensatory-mutations) selected during the adaptation-stabilization steps, draft genome sequences of the Lcn972R mutants from L. lactis L81, L62, L98, IPLA517, IPLA641, IPLA 1064 as well as the Lcn972R mutant from IPL947, L. lactis R5, were analyzed (Figure 7 and (Supplementary Table S2).

Two to seven non-synonymous mutations were detected in the Lcn972R mutants within a total of 39 genes. Among them, 23 were predicted to affect protein function according to the SIFT algorithm or a frameshift and/or a stop codon was introduced (Supplementary Table S2). Noticeably, mutants clustered together according to the parental strain. Lcn972R L. lactis from the protective culture L98 and R5 displayed an independent set of mutated genes, while nisin $\mathrm{Z}$ producers clustered together and apart from the commercial starters L. lactis L81 and L62 (Figure 7A). Taken together, these results reinforce the notion that AE-CES may lead to different outputs depending of the genetic background of the parental strain.

Six main COGs categories were overrepresented in the gene set were non-synonymous mutations were detected (Figure 7B). Not surprisingly, some mutations were found in genes involved in cell envelope biogenesis (COG M) such as murF (D-Ala-D-Ala adding enzyme), tagD1 (glycerol-3phosphate cytidylyltransferase) and pbp1 (penicillin binding protein 1A/1B) detected in IPLA517-C6, L98-C1 and E2, and L81-E2, respectively. Two genes $d l t B$ and $d l t C$ encoding functions involved in D-alanylation of the lipoteichoic acid (LTA) were also mutated in L. lactis IPLA1064-C11 and E11 and IPLA517-C6, respectively. Mutations in the genes $\operatorname{rg} p C D$, coding for a putative ATP binding cassette $(\mathrm{ABC})$ transporter likely involved in the synthesis of the polysaccharide pellicle (PSP), were also detected in the two Lcn972R mutants from L. lactis IPLA517.

Mutations in signal transduction mechanisms and transcriptional regulators were also found in several strains, anticipating a complex scenario at the transcriptional, and thus, phenotypic level. Namely, the two component system TCS-G accumulated mutations in the six Lcn972R mutants derived from the nisin $\mathrm{Z}$ producers. Other four mutants from the starters L. lactis L81 and L62 revealed mutations in ysaBC coding for a BceAB-like ABC transporter adjacent to TCS-G. Therefore, 
A

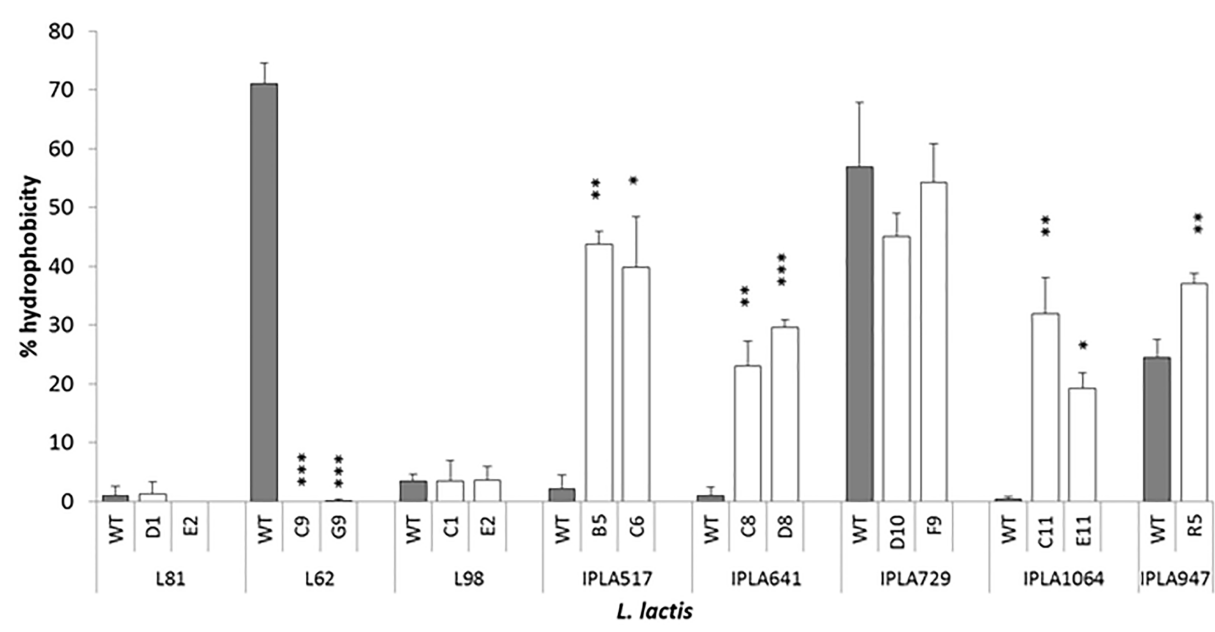

B

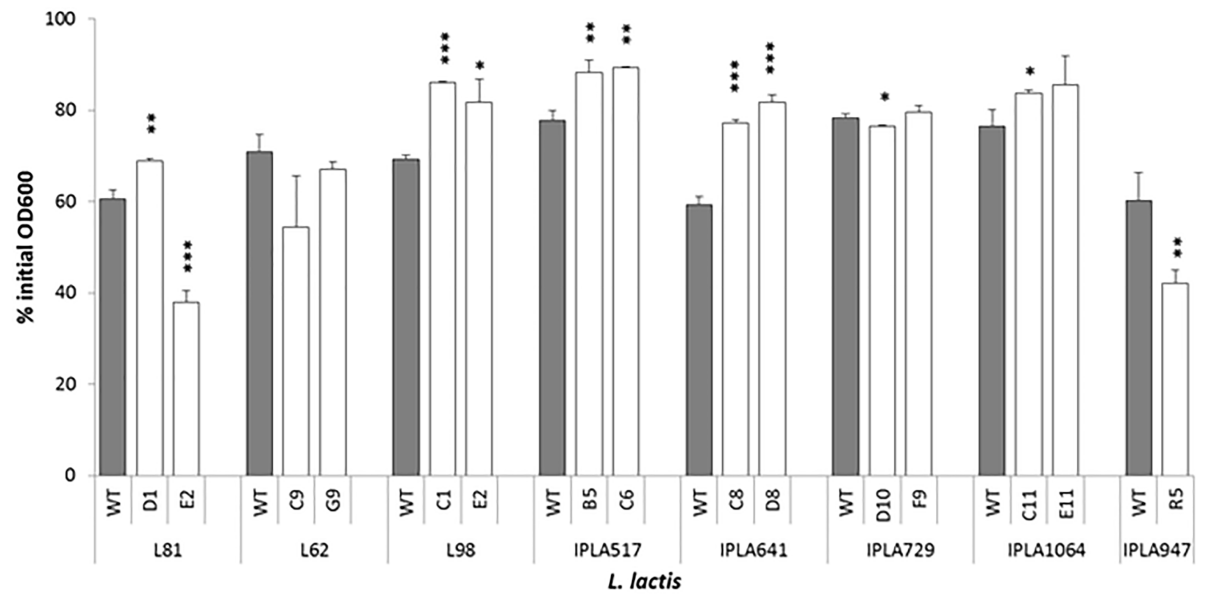

FIGURE 5 | Surface hydrophobicity (A) and autolysis (B) of L. lactis strains and their Lcn972R mutants. For autolysis, the OD 600 was measured after 150 min of incubation of cell suspensions at $30^{\circ} \mathrm{C} .{ }^{*} p<0.05,{ }^{* *} p<0.01,{ }^{* * *} p<0.001$ significantly different to the wild type strain.

the most frequent mutations among Lcn972R mutants were found in the BceAB-like TCS module which in a model organism such as Bacillus subtilis is involved in sensing and resistance to antimicrobial peptides (Ohki et al., 2003). Mutations in other stress-related genes were also found in $\mathrm{ftsH}$, coding for the membrane protease $\mathrm{FtsH}$ involved in protein quality control and regulatory functions, and in $\mathrm{relA}$, encoding the alarmone ppGpp synthetase. Nucleotide metabolism appeared to be also affected in some Lcn972R mutants. The uridylate kinase gene involved in pyrimidine metabolism ( $p y r H)$ was mutated in all the Lcn972R mutants from IPLA517, IPLA641, and IPLA1064 as well as the purine operon regulator purR in L.lactis L98-C1.

\section{DISCUSSION}

Lactic acid bacteria bacteriocins have been traditionally recognized as potent antimicrobials to be employed in food biopreservation. However, narrow-spectrum bacteriocins targeting beneficial bacteria (e.g., the dairy starter L. lactis) also have a niche of application in food biotechnology. For example, premature lysis of starter cells induced by the anti-lactococcal bacteriocins lactococcin $\mathrm{A}, \mathrm{B}$, and $\mathrm{M}$ facilitated the release of intracellular enzymes and accelerated cheese ripening (Morgan et al., 1997). In this work, we have made use of the narrow spectrum bacteriocin Lcn972 in evolution experiments with the aim of introducing diversity within L. lactis industrial starters. Our hypothesis was supported by our previous results on the characterization of Lcn972R mutants from the laboratory strain L. lactis MG1614 and the starter L. lactis IPLA947 but, in a way, this work was also inspired by the wealth of knowledge gained in the antibiotic field that clearly verified how low-level drug exposure generates genetic and phenotypic variability within pathogenic bacteria (Andersson and Hughes, 2014). On the other hand, evolution experiments have already been undertaken in L. lactis (see Bachmann et al., 2017 and references therein). These studies have demonstrated the genomic plasticity of L. lactis to fix beneficial mutations when exposed to particular conditions. 


\section{A}

L. Iactis

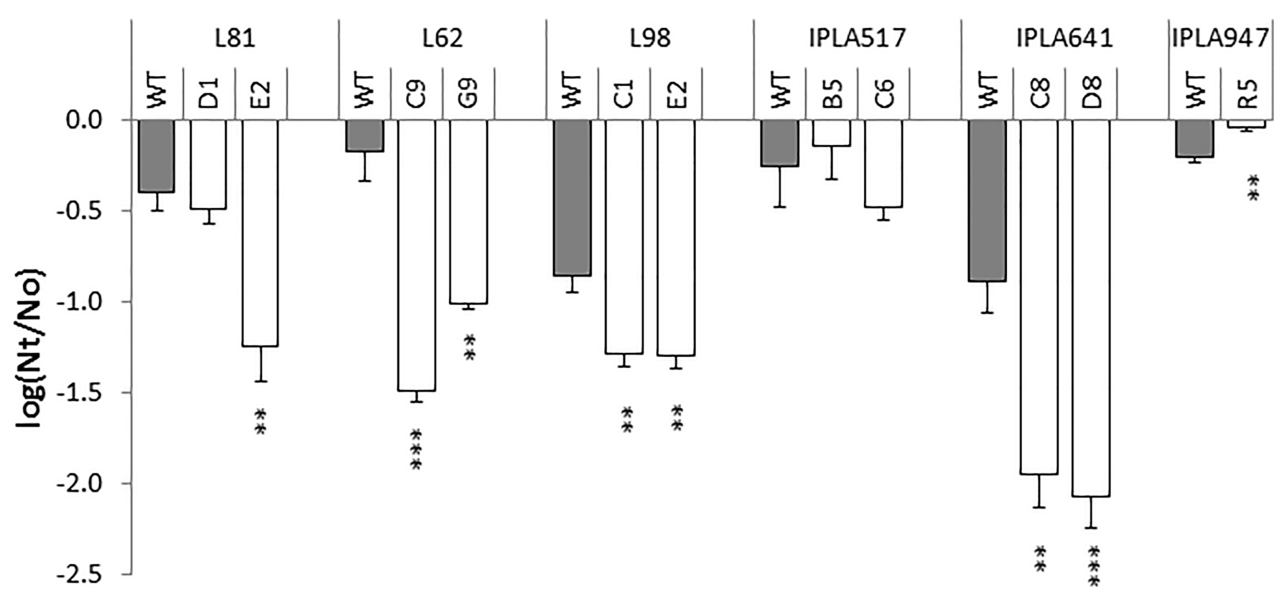

B

L. Iactis

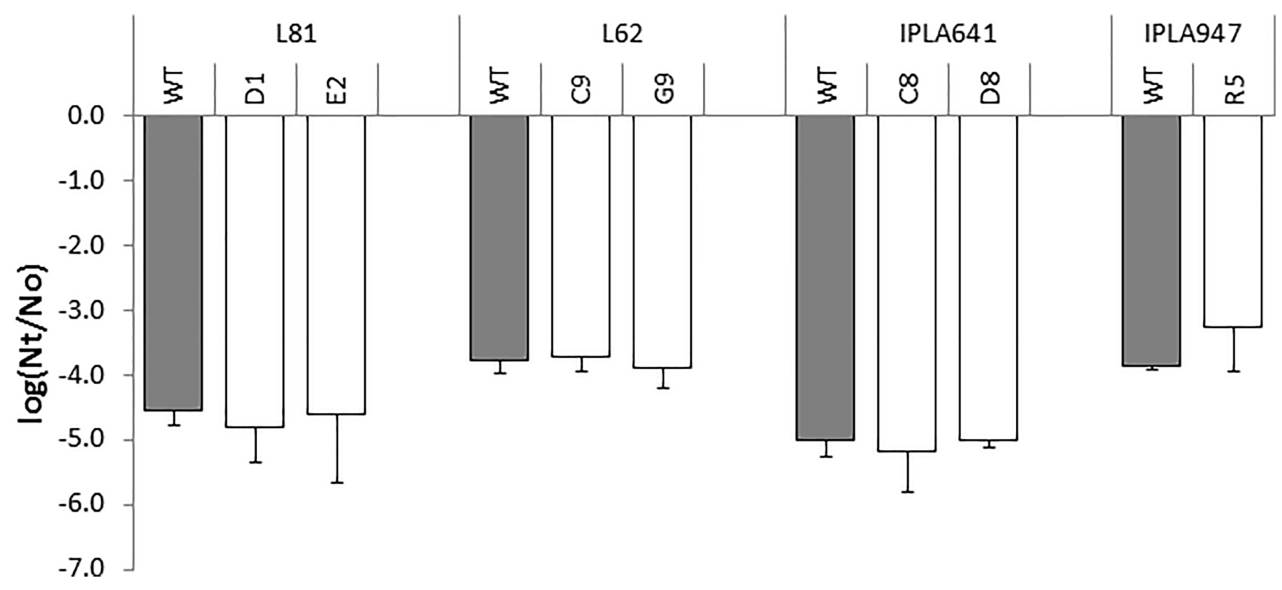

FIGURE 6 | Survival of L. lactis (gray bars) and their Lcn972R mutants (white bars) after exposure to heat (A) and low pH (B). ${ }^{*} p<0.05,{ }^{* *} p<0.01,{ }^{* * *} p<0.001$ significantly different to the wild type strain.

However, most of these studies have been carried out as proof of concept studies, using laboratory strains that differ greatly from industrial or natural L. lactis strains (Cavanagh et al., 2015; Kelleher et al., 2017).

On this basis, we have applied AE-CES to seven L. lactis strains, including commercial and isolates from raw milk cheeses, to gather information about the influence of the strain background. It was possible to select Lcn972R mutants in all cases, supporting the idea that using Lcn972 in AECES experiments could be extended to virtually all Lcn972sensitive lactococci. Moreover, in contrast to nisin resistant Lactococcus, that required the presence of nisin to retain their resistant phenotype (Kramer et al., 2006), Lcn972R mutants were stable, growth under laboratory conditions was not impaired and their resistance was not lost upon subcultivation. Two factors might have contributed to their stability. One is a possible high mutation rate in cells exposed to Lcn972. Lcn972 inhibits cell wall biosynthesis during cell division without pore formation (Martínez et al., 2008). So, cells are not instantly killed, increasing the chance to select mutations. Besides, it is known for several antibiotics, including cell wall antibiotics that mutation frequency increases when the SOS response is activated (Gutierrez et al., 2013). Although it has not been experimentally confirmed, Lcn972 seems to trigger the SOS response, as evidenced by the activation of prophages after Lcn972 treatment (Madera et al., 2009). A second factor that may have influenced the selection for stable mutants is the stabilization step after Lcn972 adaptation. Competition between clonal variants may have helped fixing beneficial compensatory mutations and the selection for fitter variants.

Aside from the physiological implications of using Lcn972 as a stressor in AE-CES, a key question was if this strategy would be compatible with the functionality of the dairy starters. The first issue we addressed was the technological 
TABLE 2 | Cross-resistance to cell wall antimicrobials and $\mathrm{NaCl}$ of L. lactis mutants resistant to Lcn972.

\begin{tabular}{|c|c|c|c|c|c|c|}
\hline L. lactis & $\begin{array}{c}\text { Bacitracin }{ }^{1} \\
(1 \mu \mathrm{g} / \mathrm{ml})\end{array}$ & $\begin{array}{l}\text { Lysozyme }^{1} \\
(0.5 \mathrm{mg} / \mathrm{ml})\end{array}$ & $\begin{array}{c}\text { Penicillin } \mathrm{G}^{1} \\
(0.1 \mu \mathrm{g} / \mathrm{ml})\end{array}$ & $\begin{array}{c}\text { Vancomycin }{ }^{1,2} \\
(0.4 \mu \mathrm{g} / \mathrm{ml})\end{array}$ & $\begin{array}{l}\text { Nisin }^{1,2} \\
(5 \mu \mathrm{g} / \mathrm{ml})\end{array}$ & $\begin{array}{r}\mathrm{NaCl}^{1} \\
(5 \%)\end{array}$ \\
\hline L81-D1 & S & $\mathrm{R}$ & S & $E$ & S & $E$ \\
\hline L81-E2 & $R$ & $\mathrm{R}$ & S & $E$ & $E$ & $E$ \\
\hline L62-C9 & $\mathrm{R}$ & $\mathrm{R}$ & S & $\mathrm{R}$ & $\mathrm{R}$ & $E$ \\
\hline L62-G9 & $\mathrm{R}$ & $\mathrm{R}$ & S & $R$ & $\mathrm{R}$ & S \\
\hline L98-C1 & $E$ & $E$ & $E$ & S & NA & $E$ \\
\hline L98-E2 & $E$ & $E$ & $E$ & S & NA & $E$ \\
\hline IPLA517-B5 & $E$ & $\mathrm{R}$ & S & $E$ & NA & S \\
\hline IPLA517-C6 & $E$ & $\mathrm{R}$ & S & $\mathrm{R}$ & NA & S \\
\hline IPLA641-C8 & S & $\mathrm{R}$ & S & $R$ & NA & S \\
\hline IPLA641-D8 & S & $\mathrm{R}$ & S & $\mathrm{R}$ & NA & S \\
\hline IPLA729-D10 & S & $\mathrm{R}$ & $E$ & $E$ & NA & $E$ \\
\hline IPLA729-F9 & $E$ & $R$ & $E$ & ND & NA & S \\
\hline IPLA1064-C11 & S & R & S & $R$ & NA & $E$ \\
\hline IPLA1064-E11 & $E$ & $E$ & $E$ & R & NA & $E$ \\
\hline IPLA947-R5 & $\mathrm{R}$ & $E$ & $E$ & ND & $\mathrm{R}$ & $S$ \\
\hline
\end{tabular}

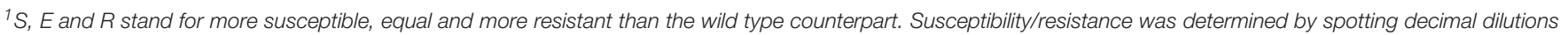
of cell suspensions on LM17 plates supplemented with the indicated concentrations.

${ }^{2} N A$, not applicable; $N D$, not determined.

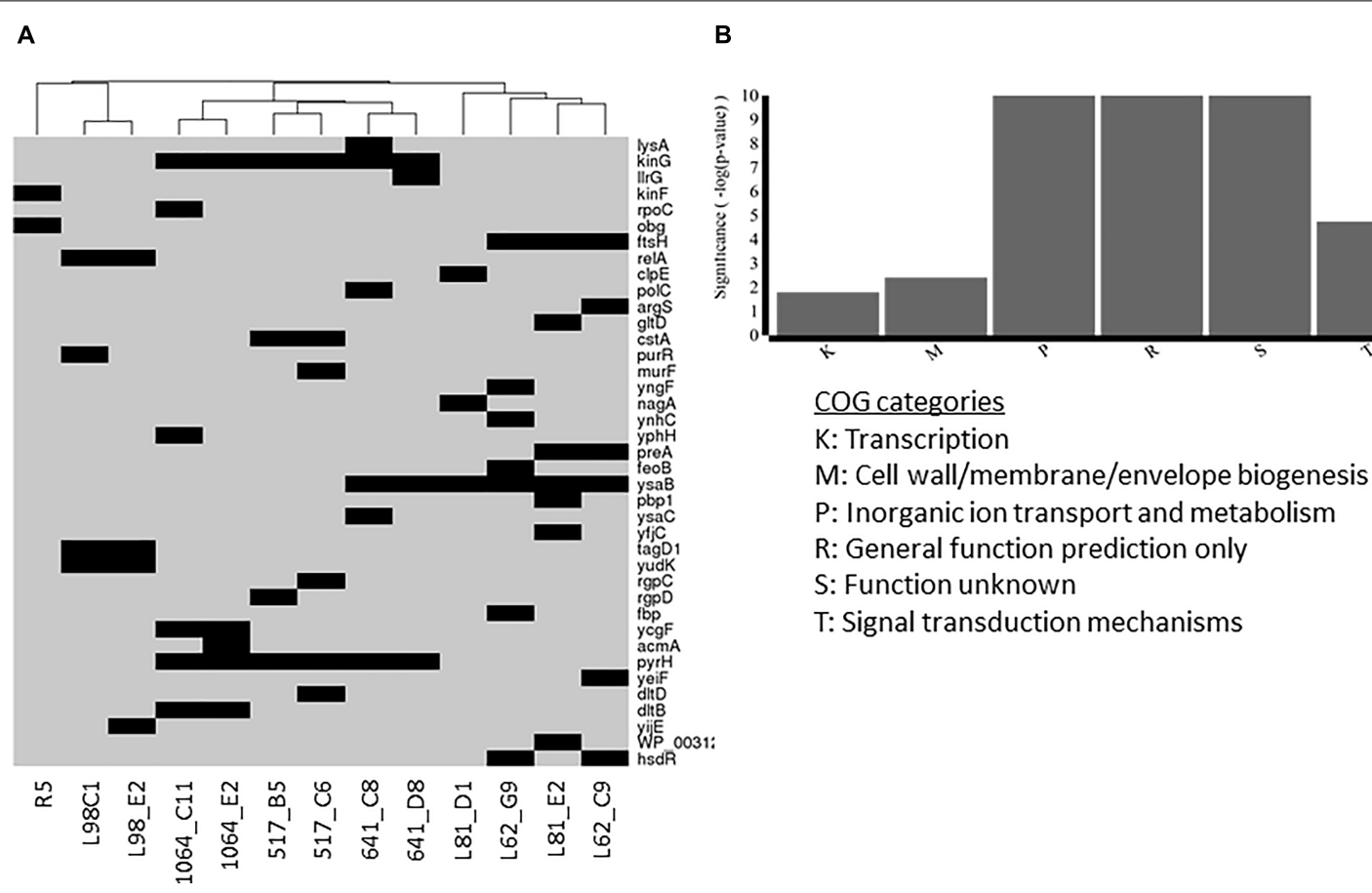

FIGURE 7 | Non-synonymous mutations present in Lcn972R L. lactis mutants (A) and functional categories overrepresented within the mutated genes (B). Presence of mutations in $(\mathbf{A})$ is represented by a black rectangle.

characterization of the Lcn972R mutants. In general, milk acidification was within the standards for dairy starters (Cogan et al., 1997) and, half of the mutants retained the same acidification rate as their corresponding WT. On the other hand, lactic acid levels were in most cases lower, suggesting a shift from homolactic to a more mixed-acid fermentation as a possible consequence of the adaptation to Lcn972.
Whether this shift results in higher acetaldehyde or diacetyl levels or not remains to be investigated. In addition, nisin production levels were not altered with the exception of the lactose negative mutants L98-C1 and E2. Taken together, the results showed that evolved mutants that retained the wild type technological phenotypes may be recovered after AE-CES. 
However, a major disadvantage encountered during AECES was the loss of plasmids in some strains. The plasmid complement of Lactococci is large and several relevant technological traits are plasmid-encoded, from lactose fermentation, proteolytic activity to bacteriophage resistance mechanisms (Ainsworth et al., 2014b). Two examples of the negative consequences of plasmid loss have been observed in this work. One was the loss of the lactose plasmid in the Lcn972R mutants from L. lactis L98 that occurred in spite of the presence of lactose as a carbon source during AE-CES. In the wild type L. lactis L98, the lactose plasmid appears to be intrinsically unstable, as described for other lactose plasmids such as pLP712 (Wegmann et al., 2012), and only lactose negative variants were selected during adaptation. The other example was the loss of a plasmid (or plasmids) encoding phage resistance mechanisms that occurred in L. lactis IPLA517 and, most likely, in the other nisin Z producing strains IPLA641 and IPLA1064. Presence of plasmid-encoded anti-phage mechanisms in the wild type strains is supported by the isolation of phage sensitive clones after plasmid curing using novobiocin, although chromosomal re-organization events might have occurred as well. Two scenarios may take place. A putative plasmid-encoded exopolysaccharide (EPS) could shield the phage receptor, as described for the lactococcal plasmid pCI658 that encodes the production of an EPS protecting Lactococcus from infection by phages phi712 and c2 (Forde and Fitzgerald, 2003). In our case, differences in the expression level of the EPS genes in LM17 and in milk may explain why no lytic plaques were observed when phages were plated on the WT strains, despite the inhibition observed in the growth inhibition test. Alternatively, loss of genes coding for abortive infection mechanisms (Abi) may also explain why Lcn972R mutants became phage sensitive and the lack of lysis plaques on otherwise "phage sensitive" L. lactis. Abi systems are altruistic phage resistant mechanisms, whereby the phage cycle is stopped and infected cells die before the phage ends its intracellular cycle (Labrie et al., 2010). Thus, progeny phages are not released, i.e., lysis plaques are not observed, and milk acidification is slowed down due to cell death. So far, searching draft genomes for putative Abi systems retrieved no relevant hits. On the contrary, a putative EPS gene cluster flanked by insertion sequences seems to be present in L. lactis IPLA517, IPLA641, and IPLA1064 and absent in their Lcn972R mutants and in the phage sensitive L. lactis IPLA729. Unfortunately, the identified contigs basically contain genes involved in EPS synthesis and the absence of any plasmid-related sequences next to the EPS genes precludes us from confirming the plasmidic nature of the EPS operon. Nevertheless, reads matching plasmid replication genes were also missing in the genomes of the Lcn972R mutants, further confirming plasmid loss in the mutants as already observed in plasmid preparations. Based on these examples, and considering that both lactose fermentation and phage resistance are key technological dairy traits, caution must be taken when applying AE-CES depending on the strains and their plasmid complement.

Further phenotypic characterization of the Lcn972R mutants, complemented by the preliminary overview of non-synonymous mutations, supports one of the outcomes of this study. That is both the inter- and intra-strain variability observed among the evolved mutants. In other words, the results emphasize the different solutions found by L. lactis to overcome the stress imposed by Lcn972. In spite of the relative low number of Lcn972R mutants characterized per strain $(n=2)$, different phenotypes were observed. This is the case, for instance, of the two L. lactis L81 Lcn972R mutants that behaved opposite to each other regarding autolysis and heat susceptibility. Moreover, as discussed below, the phenotypes selected after AE-CES also differed from those described for the laboratory strain L. lactis MG1614 (Roces et al., 2012a,b).

Changes that happened at the cell surface are exemplified by alterations on surface hydrophobicity and the autolytic behavior of the Lcn972R. Seven mutants increased their surface hydrophobicity, whereas L62-C9 and G9 lost completely the hydrophobic character of their WT. Interesting, the phage sensitive Lcn972R mutants derived from IPLA517, IPLA641, and IPLA1064 became hydrophobic, likely due to the loss of a putative hydrophilic EPS involved in phage resistance (see above). Autolysis was more uniform within Lcn972R mutants and, in general, evolved strains were less autolytic than the WT reference. These new surface properties may have a technological impact as well. It has been recently shown that the properties of $L$. lactis cell surface affects textural parameters of fermented milk (Tarazanova et al., 2018). Moreover, autolytic L. lactis may influence the development of cheese flavor (Lortal and ChapotChartier, 2005), opening an avenue for new applications of some of the evolved starters such as L. lactis L81-E2.

Considering that the cell wall is crucial for survival, one of our initial premises based on the mode of action of Lcn972 was that mutations leading to resistance to $\operatorname{Lcn} 972$ could have positive consequences on the survival to other stresses encountered during cheese manufacture. For example, extensive remodeling of the cell wall by increasing D-Asp amidation, $\mathrm{O}$-acetylation, and $\mathrm{N}$-deacetylation of the peptidoglycan promoted acid resistance in L. lactis (Hao et al., 2017; Cao et al., 2018). However, crossprotection to low $\mathrm{pH}$ was not observed within Lcn972R mutants. Furthermore, resistance to Lcn972 seems to be linked to a higher susceptibility to heat and $\mathrm{NaCl}$ (see Figure 6 and Table 2). Therefore, AE-CES using Lcn972 does not necessarily evolve robust $L$. lactis, at least, for this group of strains and the studied phenotypes. In contrast, cross-resistance to other cell wall antimicrobials was found. Increased lysozyme tolerance and cross-protection to other bacteriocins appears to be a common theme among bacteriocin resistance mutants (see Bastos et al., 2015 and references therein), regardless the mode of action, i.e., pore-forming or cell wall inhibiting bacteriocins. Likewise, sensitivity to $\beta$-lactam antibiotics is often observed (Guinane et al., 2006; Roces et al., 2012a) and our results are in line with these reports.

The preliminary overview on the non-synonymous mutations detected in the Lcn972R mutants revealed, first of all, that selected mutations seem to be dictated by the background of the parental strain. Yet, distinct intra-strain mutation profiles were observed in agreement with the notion that "in an evolutionary trajectory, multiple solutions may lead to a fitness 
increase" (Bachmann et al., 2017). These diversity may have been introduced in both adaptation and stabilization steps.

Mutations were preferentially found in genes involved in cell envelope biogenesis, active transport and regulatory functions. It should be noted that mutations that are predicted to affect protein function do not necessarily mean loss of function. Thus, the interpretation of the impact of a given mutation should be taken cautiously at this stage. With this in mind, it is fair to assume that the mutations found in the BceABlike $y s a C B$-TCS-G module, the most frequent within Lcn972R mutants, may lead to activation of this detoxification module rather than to its inactivation and, thereby, provide resistance against bacitracin, vancomycin and Lcn972 as well. Mutations in the $\mathrm{ABC}$ permease component in Streptococcus pneumoniae led to increased transcription of the $\mathrm{ABC}$ transporter genes and resistance to vancoresmycin (Becker et al., 2009). Further studies are in progress to confirm if the same applies to our Lcn972R mutants.

Another frequent mutation was found in $p y r H$, encoding the UMP kinase involved in the last steps of the synthesis of RNA precursors. This mutation may have direct consequences on the architecture of the cell wall and turn cells resistant to cell wall antimicrobials as lysozyme and Lcn972. Recently, mutations in $p y r B$, coding for the aspartate carbamoyltransferase in pyrimidine metabolism, were frequently found in L. lactis resistance to lysozyme (Solopova et al., 2016). The authors claimed that limiting the availability of L-Asp for nucleotide biosynthesis results in a highly cross-linked and rigid peptidoglycan that interferes with lysozyme activity. Other mutations that may have an impact on surface properties are those found in the mutants from IPLA517 in the genes $r g p C D$ located in the cell wall polysaccharide gene cluster. These genes putatively encoded the $\mathrm{ABC}$ transporter of the polysaccharide precursors and, when non-functional, the amount of the polysaccharide pellicle may be reduced, increasing surface hydrophobicity. This cell wall polysaccharide is a well-stablished receptor for several L. lactis phages of the 936 and P335 groups (Ainsworth et al., 2014a). Unfortunately, none of the phages able to infect the WT L. lactis IPLA517 belong to any of these phage groups and it could not be established if these mutations may render IPLA517 less prone to phage attack. Finally, absence of growth in milk of L. lactis L98-C1 could be linked to the mutation in purR. The inactivation of this transcriptional activator would potentially render the mutant unable of de novo synthesis of purines required for growth in milk, a substrate low in purine content (Kilstrup and Martinussen, 1998). Interestingly, mutations connected to purine nucleotide metabolism are often behind multi-stress resistance in L. lactis (Ryssel et al., 2014) which may explain the selection of such mutations during AE-CES.

\section{CONCLUSION}

In this work we have identified advantages and disadvantages of applying AE-CES in L. lactis and the consequences for its performance as a dairy starter. AE-CES appears as a feasible strategy to introduce phenotypic and genetic diversity in L. lactis, regardless of the strain origin. Evolved strains may retain similar technological traits as the strains they are derived from, while acquiring new ones. Plasmid loss was one of the main disadvantages that might be overcome by combining AE-CES with conditions for selection of the plasmid of interest or by screening a larger number of evolved clones. Although still preliminary, data gathered from draft genomes anticipates the likely selection for mutations activating detoxification modules and changes at the cell surface which may have practical implications in milk fermentations. The phenotypic and genetic characterization of the evolved strains has also emphasized the plasticity of $L$. lactis to give rise to new phenotypes, providing the versatility required for adaptive evolution to become an excellent tool in strain development programs.

\section{DATA AVAILABILITY}

Datasets are available on request. The raw data supporting the conclusions of this manuscript will be made available by the authors, without undue reservation, to any qualified researcher.

\section{AUTHOR CONTRIBUTIONS}

ML-G ran the evolution experiments. ML-G, TJ, and BM performed the phenotypic characterization experiments. SE, TJ, and BM analyzed mutations. AR, AN, TJ, and BM conceived and designed the study. All authors were involved in analysis of the results and drafting the manuscript prior to submission. Tasks were supervised by AR and BM at IPLA-CSIC and by AN at Chr. Hansen A/S.

\section{FUNDING}

This work was funded by grants BIO2013-46266-R and BIO201788147-R (Ministerio de Economía y Competitividad, Spain). Activities of the DairySafe group at IPLA-CSIC were also funded by GRUPIN14-139 (FEDER funds and program of Science, Technology and Innovation 2013-2017, Principado de Asturias, Spain).

\section{ACKNOWLEDGMENTS}

We thank Esther Sánchez Llana (IPLA-CSIC, Spain) for her technical assistance with plasmid isolation and phage experiments. We acknowledge support of the publication fee by the CSIC Open Access Publication Support Initiative through its Unit of Information Resources for Research (URICI).

\section{SUPPLEMENTARY MATERIAL}

The Supplementary Material for this article can be found online at: https://www.frontiersin.org/articles/10.3389/fmicb.2018. 02654/full\#supplementary-material 


\section{REFERENCES}

Ainsworth, S., Sadovskaya, I., Vinogradov, E., Courtin, P., Guerardel, Y., Mahony, J., et al. (2014a). Differences in lactococcal cell wall polysaccharide structure are major determining factors in bacteriophage sensitivity. mBio 5 , e880-e814. doi: 10.1128/mBio.00880-14

Ainsworth, S., Stockdale, S., Bottacini, F., Mahony, J., and Van Sinderen, D. (2014b). The Lactococcus lactis plasmidome: much learnt, yet still lots to discover. FEMS Microbiol. Rev. 38, 1066-1088. doi: 10.1111/1574-6976.12074

Álvarez-Sieiro, P., Montalbán-López, M., Mu, D., and Kuipers, O. P. (2016). Bacteriocins of lactic acid bacteria: extending the family. Appl. Microbiol. Biotechnol. 100, 2939-2951. doi: 10.1007/s00253-016-7343-9

Andersson, D. I., and Hughes, D. (2014). Microbiological effects of sublethal levels of antibiotics. Nat. Rev. Microbiol. 12, 465-478. doi: 10.1038/nrmicro3270

Babicki, S., Arndt, D., Marcu, A., Liang, Y., Grant, J. R., Maciejewski, A., et al. (2016). Heatmapper: web-enabled heat mapping for all. Nucleic Acids Res. 44, W147-W153. doi: 10.1093/nar/gkw419

Bachmann, H., Molenaar, D., Branco Dos Santos, F., and Teusink, B. (2017). Experimental evolution and the adjustment of metabolic strategies in lactic acid bacteria. FEMS Microbiol. Rev. 41, S201-S219. doi: 10.1093/femsre/fux024

Bachmann, H., Starrenburg, M. J., Molenaar, D., Kleerebezem, M., and Van Hylckama Vlieg, J. E. (2012). Microbial domestication signatures of Lactococcus lactis can be reproduced by experimental evolution. Genome Res. 22, 115-124. doi: 10.1101/gr.121285.111

Bastos M. C., Coelho, M. L., and Santos, O. C. (2015). Resistance to bacteriocins produced by Gram-positive bacteria. Microbiology 161, 683-700. doi: 10.1099/ mic.0.082289-0

Becker, P., Hakenbeck, R., and Henrich, B. (2009). An ABC transporter of Streptococcus pneumoniae involved in susceptibility to vancoresmycin and bacitracin. Antimicrob. Agents Chemother. 53, 2034-2041. doi: 10.1128/AAC. 01485-08

Brettin, T., Davis, J. J., Disz, T., Edwards, R. A., Gerdes, S., Olsen, G. J., et al. (2015). RASTtk: a modular and extensible implementation of the RAST algorithm for building custom annotation pipelines and annotating batches of genomes. Sci. Rep. 5:8365. doi: 10.1038/srep08365

Cao, L., Liang, D., Hao, P., Song, Q., Xue, E., Caiyin, Q., et al. (2018). The increase of $\mathrm{O}$-acetylation and $\mathrm{N}$-deacetylation in cell wall promotes acid resistance and nisin production through improving cell wall integrity in Lactococcus lactis. J. Ind. Microbiol. Biotechnol. 45, 813-825. doi: 10.1007/s10295-018-2052-2

Cárcoba, R., Delgado, T., and Rodríguez, A. (2000). Comparative performance of a mixed strain starter in cow's milk, ewe's milk and mixtures of these milks. Eur. Food Res. Technol. 211, 141-146. doi: 10.1007/s002170000157

Cavanagh, D., Casey, A., Altermann, E., Cotter, P. D., Fitzgerald, G. F., and Mcauliffe, O. (2015). Evaluation of Lactococcus lactis isolates from nondairy sources with potential dairy applications reveals extensive phenotype-genotype disparity and implications for a revised species. Appl. Environ. Microbiol. 81, 3961-3972. doi: 10.1128/AEM.04092-14

Chapot-Chartier, M. P., and Kulakauskas, S. (2014). Cell wall structure and function in lactic acid bacteria. Microb. Cell Fact. 13(Suppl. 1):S9. doi: 10.1186/ 1475-2859-13-S1-S9

Chen, J., Shen, J., Ingvar Hellgren, L., Ruhdal Jensen, P., and Solem, C. (2015). Adaptation of Lactococcus lactis to high growth temperature leads to a dramatic increase in acidification rate. Sci. Rep. 5:14199. doi: 10.1038/srep14199

Cogan, T. M., Barbosa, M., Beuvier, E., Bianchi-Salvadori, B., Cocconcelli, P. S., Fernandes, I., et al. (1997). Characterization of the lactic acid bacteria in artisanal dairy products. J. Dairy Res. 64, 409-421. doi: 10.1017/ S0022029997002185

Derkx, P. M., Janzen, T., Sørensen, K. I., Christensen, J. E., Stuer-Lauridsen, B., and Johansen, E. (2014). The art of strain improvement of industrial lactic acid bacteria without the use of recombinant DNA technology. Microb. Cell Fact. 13(Suppl. 1):S5. doi: 10.1186/1475-2859-13-S1-S5

Forde, A., and Fitzgerald, G. F. (2003). Molecular organization of exopolysaccharide (EPS) encoding genes on the lactococcal bacteriophage adsorption blocking plasmid, pCI658. Plasmid 49, 130-142. doi: 10.1016/S0147-619X(02)00156-7

Garneau, J. E., and Moineau, S. (2011). Bacteriophages of lactic acid bacteria and their impact on milk fermentations. Microb. Cell Fact. 10(Suppl. 1):S20. doi: 10.1186/1475-2859-10-S1-S20
Guinane, C. M., Cotter, P. D., Hill, C., and Ross, R. P. (2006). Spontaneous resistance in Lactococcus lactis IL1403 to the lantibiotic lacticin 3147. FEMS Microbiol. Lett. 260, 77-83. doi: 10.1111/j.1574-6968.2006.00302.x

Gutierrez, A., Laureti, L., Crussard, S., Abida, H., Rodriguez-Rojas, A., Blazquez, J., et al. (2013). beta-lactam antibiotics promote bacterial mutagenesis via an RpoS-mediated reduction in replication fidelity. Nat. Commun. 4:1610. doi: $10.1038 /$ ncomms 2607

Hao, P., Liang, D., Cao, L., Qiao, B., Wu, H., Caiyin, Q., et al. (2017). Promoting acid resistance and nisin yield of Lactococcus lactis F44 by genetically increasing D-Asp amidation level inside cell wall. Appl. Microbiol. Biotechnol. 101, 61376153. doi: 10.1007/s00253-017-8365-7

Johansen, E. (2018). Use of natural selection and evolution to develop new starter cultures for fermented foods. Annu. Rev. Food Sci. Technol. 9, 411-428. doi: 10.1146/annurev-food-030117-012450

Johansen, E., Øregaard, G., Sørensen, K. I., and Derkx, P. M. F. (2015). “10 - Modern approaches for isolation, selection, and improvement of bacterial strains for fermentation applications," in Advances in Fermented Foods and Beverages, ed. W. Holzapfel (Cambridge: Elsevier), 227-248.

Johnson, M. E. (2017). A 100-year review: cheese production and quality. J. Dairy Sci. 100, 9952-9965. doi: 10.3168/jds.2017-12979

Kelleher, P., Bottacini, F., Mahony, J., Kilcawley, K. N., and Van Sinderen, D. (2017). Comparative and functional genomics of the Lactococcus lactis taxon; insights into evolution and niche adaptation. BMC Genomics 18:267. doi: 10. 1186/s12864-017-3650-5

Kilstrup, M., and Martinussen, J. (1998). A transcriptional activator, homologous to the Bacillus subtilis PurR repressor, is required for expression of purine biosynthetic genes in Lactococcus lactis. J. Bacteriol. 180, 3907-3916.

Kjos, M., Oppegård, C., Diep, D. B., Nes, I. F., Veening, J. W., Nissen-Meyer, J., et al. (2014). Sensitivity to the two-peptide bacteriocin lactococcin G is dependent on UppP, an enzyme involved in cell-wall synthesis. Mol. Microbiol. 92, 1177-1187. doi: $10.1111 / \mathrm{mmi} .12632$

Kramer, N. E., Hasper, H. E., Van Den Bogaard, P. T., Morath, S., De Kruijff, B., Hartung, T., et al. (2008). Increased D-alanylation of lipoteichoic acid and a thickened septum are main determinants in the nisin resistance mechanism of Lactococcus lactis. Microbiology 154, 1755-1762. doi: 10.1099/mic.0.2007/ 015412-0

Kramer, N. E., Van Hijum, S. A., Knol, J., Kok, J., and Kuipers, O. P. (2006). Transcriptome analysis reveals mechanisms by which Lactococcus lactis acquires nisin resistance. Antimicrob. Agents Chemother. 50, 1753-1761. doi: 10.1128/AAC.50.5.1753-1761.2006

Kristo, E., Biliaderis, C. G., and Tzanetakis, N. (2003). Modelling of the acidification process and rheological properties of milk fermented with a yogurt starter culture using response surface methodology. Food Chem. 83, 437-446. doi: 10.1016/S0308-8146(03)00126-2

Labrie, S. J., Samson, J. E., and Moineau, S. (2010). Bacteriophage resistance mechanisms. Nat. Rev. Microbiol. 8, 317-327. doi: 10.1038/nrmicro2315

López-González, M. J., Campelo, A. B., Picon, A., Rodríguez, A., and Martínez, B. (2018). Resistance to bacteriocin Lcn972 improves oxygen tolerance of Lactococcus lactis IPLA947 without compromising its performance as a dairy starter. BMC Microbiol. 18:76. doi: 10.1186/s12866-018-1222-8

Lortal, S., and Chapot-Chartier, M. P. (2005). Role, mechanisms and control of lactic acid bacteria lysis in cheese. Int. Dairy J. 15, 857-871. doi: 10.1016/j. idairyj.2004.08.024

Madera, C., García, P., Rodríguez, A., Suárez, J. E., and Martínez, B. (2009). Prophage induction in Lactococcus lactis by the bacteriocin Lactococcin 972. Int. J. Food Microbiol. 129, 99-102. doi: 10.1016/j.ijfoodmicro.2008. 11.004

Martínez, B., Böttiger, T., Schneider, T., Rodríguez, A., Sahl, H. G., and Wiedemann, I. (2008). Specific interaction of the unmodified bacteriocin Lactococcin 972 with the cell wall precursor lipid II. Appl. Environ. Microbiol. 74, 4666-4670. doi: 10.1128/AEM.00092-08

Martínez, B., Suárez, J. E., and Rodríguez, A. (1995). Antimicrobials produced by wild lactococcal strains isolated from homemade cheeses. J. Food Protect. 58, 1118-1123. doi: 10.4315/0362-028X-58.10.1118

Martínez, B., Zomer, A. L., Rodríguez, A., Kok, J., and Kuipers, O. P. (2007). Cell envelope stress induced by the bacteriocin Lcn 972 is sensed by the lactococcal two-component system CesSR. Mol. Microbiol. 64, 473-486. doi: 10.1111/j. 1365-2958.2007.05668.x 
McAuliffe, O. (2018). Symposium review: Lactococcus lactis from nondairy sources: their genetic and metabolic diversity and potential applications in cheese. J. Dairy Sci. 101, 3597-3610. doi: 10.3168/jds.2017-13331

Meyrand, M., Boughammoura, A., Courtin, P., Mezange, C., Guillot, A., and Chapot-Chartier, M. P. (2007). Peptidoglycan N-acetylglucosamine deacetylation decreases autolysis in Lactococcus lactis. Microbiology 153, 32753285. doi: 10.1099/mic.0.2007/005835-0

Miljkovic, M., Uzelac, G., Mirkovic, N., Devescovi, G., Diep, D. B., Venturi, V., et al. (2016). LsbB bacteriocin interacts with the third transmembrane domain of the YvjB receptor. Appl. Environ. Microbiol. 82, 5364-5374. doi: 10.1128/ AEM.01293-16

Morgan, S., Ross, R. P., and Hill, C. (1997). Increasing starter cell lysis in Cheddar cheese using a bacteriocin-producing adjunct. J. Dairy Sci. 80, 1-10. doi: 10. 3168/jds.S0022-0302(97)75906-X

Ohki, R., Giyanto, G., Tateno, K., Masuyama, W., Moriya, S., Kobayashi, K., et al. (2003). The BceRS two-component regulatory system induces expression of the bacitracin transporter, BceAB, in Bacillus subtilis. Mol. Microbiol. 49, 1135-1144. doi: 10.1046/j.1365-2958.2003.03653.x

O'sullivan, D. J., and Klaenhammer, T. R. (1993). Rapid mini-prep isolation of high-quality plasmid DNA from Lactococcus and Lactobacillus spp. Appl. Environ. Microbiol. 59, 2730-2733.

Roces, C., Courtin, P., Kulakauskas, S., Rodríguez, A., Chapot-Chartier, M. P., and Martínez, B. (2012a). Isolation of Lactococcus lactis mutants simultaneously resistant to the cell wall-active bacteriocin Lcn972, lysozyme, nisin and bacteriophage c2. Appl. Environ. Microbiol. 78, 4157-4163. doi: 10.1128/AEM. 00795- 12

Roces, C., Pérez, V., Campelo, A. B., Blanco, D., Kok, J., Kuipers, O. P., et al. (2012b). The putative lactococcal extracytoplasmic function anti-sigma factor llmg2447 determines resistance to the cell wall-active bacteriocin lcn972. Antimicrob. Agents Chemother. 56, 5520-5527. doi: 10.1128/AAC. 01206-12

Rodríguez, A., Martínez, B., and Suárez, J. E. (2012). “Dairy starter cultures”, in Handbook of Animal- Based Fermented Food and Beverages, Second Edn, ed. Y. H. Hui (Boca Raton, FL: CRC press), 31-48.

Ryssel, M., Hviid, A. M., Dawish, M. S., Haaber, J., Hammer, K., Martinussen, J., et al. (2014). Multi-stress resistance in Lactococcus lactis is actually escape from purine-induced stress sensitivity. Microbiology 160, 2551-2559. doi: 10.1099/ mic. $0.082586-0$
Solopova, A., Formosa-Dague, C., Courtin, P., Furlan, S., Veiga, P., Pechoux, C., et al. (2016). Regulation of cell wall plasticity by nucleotide metabolism in Lactococcus lactis. J. Biol. Chem. 291, 11323-11336. doi: 10.1074/jbc.M116. 714303

Sørensen, K. I., Curic-Bawden, M., Junge, M. P., Janzen, T., and Johansen, E. (2016). Enhancing the sweetness of yoghurt through metabolic remodeling of carbohydrate metabolism in Streptococcus thermophilus and Lactobacillus delbrueckii subsp. bulgaricus. Appl. Environ. Microbiol. 82, 3683-3692. doi: 10.1128/AEM.00462-16

Steen, A., Palumbo, E., Deghorain, M., Cocconcelli, P. S., Delcour, J., Kuipers, O. P., et al. (2005). Autolysis of Lactococcus lactis is increased upon D-alanine depletion of peptidoglycan and lipoteichoic acids. J. Bacteriol. 187, 114-124. doi: 10.1128/JB.187.1.114-124.2005

Tarazanova, M., Huppertz, T., Kok, J., and Bachmann, H. (2018). Altering textural properties of fermented milk by using surface-engineered Lactococcus lactis. Microb. Biotechnol. 11, 770-780. doi: 10.1111/1751-7915.13278

Tessema, G. T., Moretro, T., Snipen, L., Axelsson, L., and Naterstad, K. (2011). Global transcriptional analysis of spontaneous sakacin P-resistant mutant strains of Listeria monocytogenes during growth on different sugars. PLoS One 6:e16192. doi: 10.1371/journal.pone.0016192

Wegmann, U., Overweg, K., Jeanson, S., Gasson, M., and Shearman, C. (2012). Molecular characterization and structural instability of the industrially important composite metabolic plasmid pLP712. Microbiology 158, 2936-2945. doi: $10.1099 /$ mic. $0.062554-0$

Conflict of Interest Statement: AN and TJ are employees of Chr. Hansen A/S.

The remaining authors declare that the research was conducted in the absence of any commercial or financial relationships that could be construed as a potential conflict of interest.

Copyright (c) 2018 López-González, Escobedo, Rodríguez, Neves, Janzen and Martinez. This is an open-access article distributed under the terms of the Creative Commons Attribution License (CC BY). The use, distribution or reproduction in other forums is permitted, provided the original author(s) and the copyright owner(s) are credited and that the original publication in this journal is cited, in accordance with accepted academic practice. No use, distribution or reproduction is permitted which does not comply with these terms. 